\title{
elF3 and Its mRNA-Entry-Channel Arm Contribute to the Recruitment of mRNAs With Long $5^{\prime}$-Untranslated Regions
}

\begin{abstract}
Andrei Stanciu ${ }^{1}$, Juncheng Luo ${ }^{2}$, Lucy Funes ${ }^{3}$, Shanya Galbokke Hewage ${ }^{3}$, Shardul D. Kulkarni ${ }^{4}$ and Colin Echeverría Aitken ${ }^{2,3 *}$
\end{abstract}

${ }^{1}$ Computer Science Department, Vassar College, Poughkeepsie, NY, United States, ${ }^{2}$ Biochemistry Program, Vassar College, Poughkeepsie, NY, United States, ${ }^{3}$ Biology Department, Vassar College, Poughkeepsie, NY, United States, ${ }^{4}$ Department of Biochemistry and Molecular Biology, Penn State Eberly College of Medicine, University Park, PA, United States

Translation initiation in eukaryotes is a multi-step pathway and the most regulated phase of translation. Eukaryotic initiation factor 3 (elF3) is the largest and most complex of the translation initiation factors, and it contributes to events throughout the initiation pathway. In particular, elF3 appears to play critical roles in mRNA recruitment. More recently, elF3 has been implicated in driving the selective translation of specific classes of mRNAs. However, unraveling the mechanism of these diverse contributions-and disentangling the roles of the individual subunits of the elF3 complex-remains challenging. We employed ribosome profiling of budding yeast cells expressing two distinct mutations targeting the elF3 complex. These mutations either disrupt the entire complex or subunits positioned near the mRNA-entry channel of the ribosome and which appear to relocate during or in response to mRNA binding and start-codon recognition. Disruption of either the entire elF3 complex or specific targeting of these subunits affects $m R N A s$ with long $5^{\prime}$-untranslated regions and whose translation is more dependent on elF4A, elF4B, and Ded1 but less dependent on elF4G, elF4E, and PABP. Disruption of the entire elF3 complex further affects mRNAs involved in mitochondrial processes and with structured $5^{\prime}$-untranslated regions. Comparison of the suite of mRNAs most sensitive to both mutations with those uniquely sensitive to disruption of the entire complex sheds new light on the specific roles of individual subunits of the elF3 complex.

Keywords: elF3, translation initiation, translational regulation, mRNA recruitment, ribosome, ribosome profiling, ribo-seq

\section{INTRODUCTION}

Accepted: 13 December 2021

Published: 11 January 2022

Citation:

Stanciu A, Luo J, Funes L, Galbokke Hewage S, Kulkarni SD and Aitken CE (2022) elF3 and Its mRNAEntry-Channel Arm Contribute to the Recruitment of mRNAs With Long $5^{\prime}$ Untranslated Regions.

Front. Mol. Biosci. 8:787664. doi: 10.3389/fmolb.2021.787664
Translation initiation is the rate-limiting and most regulated phase of translation (Sonenberg and Hinnebusch, 2009; Jackson et al., 2010). Translation initiation in eukaryotes requires the ribosome-the macromolecular machine responsible for synthesizing the proteins encoded by messenger RNA molecules in all kingdoms of life-to dock at the very $5^{\prime}$ end of a mRNA molecule and then scan to identify the start codon for translation, usually the first AUG. The sequence through which the ribosome must scan, known either as the $5^{\prime}$-untranslated region $\left(5^{\prime}\right.$ UTR) or the transcript leader (TL), can be in excess of a 1,000 nucleotides in length and contain regions of defined secondary structure or upstream open reading frames (uORFS) demarcated by 
either cognate (AUG) or near-cognate start codons and whose translation can regulate translation of the downstream open reading frame (ORF) (Hinnebusch et al., 2016).

At least twelve protein initiation factors (eIFs) collaborate with the ribosome to facilitate its navigation of these obstacles (Aitken and Lorsch, 2012; Shivaya Valasek, 2012; Hinnebusch, 2014; Hinnebusch, 2017). The process begins with the formation of a pre-initiation complex (PIC) comprising the small (40S) ribosomal subunit, a ternary complex (TC) of the initiator methionyl tRNA $\left(\mathrm{tRNA}_{\mathrm{i}}\right)$, the GTPase eIF2, and GTP $\left(\mathrm{tRNA}_{\mathrm{i}} \bullet \mathrm{eIF} 2 \bullet \mathrm{GTP}\right)$, and the initiation factors eIF1, eIF1A, eIF5, and eIF3. The PIC then docks at the $5^{\prime}$ end of the mRNA in collaboration with the eIF4F complex comprising the cap-binding protein eIF4E, the scaffolding protein eIF4G, and the helicase eIF4A, which may facilitate initial docking of the PIC by relaxing structural complexity near the $5^{\prime}$ end of the mRNA (Yourik et al., 2017). Once docked at the $5^{\prime}$ end, the PIC scans in the $3^{\prime}$ direction to identify the start codon. Scanning is thought to be facilitated by eIF4A and eIF4B, which binds the $40 \mathrm{~S}$ subunit (Walker et al., 2013). The helicase Ded1 also plays an important role in scanning, perhaps by resolving defined structural elements within the 5' UTR that might otherwise prevent efficient scanning (Gupta et al., 2018).

The largest and most complex of the initiation factors is eIF3, a multi-subunit complex comprising at least 5 essential subunits in the yeast $S$. cerevisiae and at least 12 subunits in mammalian cells (Hinnebusch, 2006; Valášek et al., 2017). eIF3 participates in every component step of translation initiation. It stabilizes and promotes formation of the PIC via interactions with the $40 \mathrm{~S}$ subunit, eIF1, and eIF2 within the TC (Asano et al., 2000; Valášek et al., 2002; Majumdar et al., 2003; Valášek et al., 2003; Nielsen et al., 2006; Sokabe and Fraser, 2014). eIF3 is also required, both in vivo and in vitro, for mRNA recruitment by the PIC (Jivotovskaya et al., 2006; Mitchell et al., 2010; Aitken et al., 2016), a process consisting of PIC docking, scanning, and startcodon recognition. Consistent with this role, eIF3 binds the PIC at the solvent face but projects appendages near both the mRNAentry and mRNA-exit channels of the ribosome (Aylett et al., 2015; Des Georges et al., 2015; Llácer et al., 2018). At the mRNAexit channel, the eIF3a subunit (and eIF3d in higher eukaryotes) appears to interact functionally or physically with the mRNA (Szamecz et al., 2008; Munzarová et al., 2011) and the very $\mathrm{N}$-terminal region of eIF3a seems to stabilize the binding of mRNA to the PIC (Aitken et al., 2016). Subunits of the human eIF3 complex bind to eIF4G (Villa et al., 2013), and some of these were found interacting directly with components of the eIF4F complex at the mRNA-exit channel in a recent high-resolution structure of the human 48S PIC (Querido et al., 2020).

Near the mRNA-entry channel, the C-terminal domain (CTD) of eIF3a interacts with $40 \mathrm{~S}$ elements that mediate the transition between the open (docking- and scanning-competent) and closed (scanning-arresting) conformations of the PIC (Chiu et al., 2010; Dong et al., 2017). High-resolution structural models of eIF3 bound to the PIC reveal that the eIF3a CTD, eIF3b, eIF3i, and eIF3g compose this mRNA-entry-channel arm (Des Georges et al., 2015; Simonetti et al., 2016; Llácer et al., 2018). Moreover, structural models of the PIC either lacking or bound to mRNA reveal distinct positions of this arm (Llácer et al., 2015; Llácer et al., 2018). In the absence of mRNA, these subunits are found bound to the solvent face of the PIC; in the presence of mRNA, but prior to start-codon recognition, the mRNA-entry-channel arm is found at the intersubunit face but then appears to relocate to its original position at the solvent face upon start-codon recognition. Together with the observations that mutations to the eIF3a CTD (Valášek et al., 2002; Chiu et al., 2010), eIF3b (Nielsen et al., 2004; Elantak et al., 2010), eIF3i (Herrmannová et al., 2012), and eIF3g (Cuchalova et al., 2010) elicit phenotypes consistent with defects in the component events of mRNA recruitment and affect the kinetics of mRNA recruitment in vitro (Aitken et al., 2016), this suggests that the eIF3 mRNA-entry-channel arm, and its potential repositioning in response to mRNA binding and start-codon recognition, may play an important mechanistic role in mRNA recruitment.

To investigate the role of eIF3 and components of the eIF3 mRNA-entry-channel arm in mRNA recruitment and its component events, we employed ribosome profiling (Ingolia et al., 2009) to follow the repercussions of specific eIF3 mutations on the translational efficiency (TE) of mRNAs across the transcriptome. By comparing the features of mRNAs most sensitive to each mutation with those least sensitive to these mutations, we shed new light on the role of eIF3 and its mRNAentry-channel arm in mRNA recruitment. This approach has previously been employed to illuminate the transcriptome-scale role of several initiation factors, including eIF1 (Zhou et al., 2020), eIF1A (Martin-Marcos et al., 2017), eIF4A (Sen et al., 2015), eIF4B (Sen et al., 2016), and Ded1 (Sen et al., 2019). Here, we focused on two mutations whose effects on translation initiation have been previously explored with both genetic and biochemical tools. The first of these mutants-tif32 $2^{t d} /$ prt $^{\text {td }}$ (eIF3a/b Degron)expresses temperature sensitive degron $(\mathrm{td})$ alleles of the eIF3a (TIF32) and eIF3b (PRT1) subunits (Jivotovskaya et al., 2006). Growth of this strain under restrictive conditions results in the depletion of eIF3a and eIF3b. This in turn disrupts the entire eIF3 complex, mimicking an eIF3 deletion mutant under these conditions. This disruption of the eIF3 complex further interferes with mRNA binding and $48 \mathrm{~S}$ formation by the PIC, providing evidence for the role of eIF3 in mRNA recruitment (Jivotovskaya et al., 2006). The second mutation we investigated is a mutation to eIF3i that abrogates eIF3i binding to eIF3b: eIF3i DDKK (Herrmannová et al., 2012). Because the binding of both eIF3i and eIF3g to the eIF3 complex depends on this interaction, the eIF3i DDKK mutation mimics the absence of both subunits; purification of eIF3 from eIF3i DDKK cells via tagged eIF3b yields the wild-type $\mathrm{a} / \mathrm{b} / \mathrm{c}$ sub-complex (Aitken et al., 2016). The eIF3i DDKK mutation was previously shown to interfere with scanning and start-codon recognition in vivo and in cell extracts (Herrmannová et al., 2012). In addition, subsequent in vitro investigation demonstrated that, in the absence of eIF3i and eIF3g, the eIF3 a/b/c sub-complex is unable to promote recruitment of a natural, capped mRNA (Aitken et al., 2016).

By investigating the effects of these two mutations-which mimic the loss of either the entire eIF3 complex or two subunits of the eIF3 mRNA-entry-channel arm-we hoped to disentangle the roles of eIF3i and eIF3g from that of the entire eIF3 complex. 
In the presence of both mutations, we observed strong decreases in global translation levels and were able to identify mRNAs whose relative translational efficiency is either more or less sensitive-as compared to the total population of mRNAs-to each mutation. By comparing the features of these mRNAs with each other and with mRNAs sensitive to mutations targeting other initiation factors, we shed further light on the roles of eIF3 and its mRNA-entry-channel arm during mRNA recruitment. Contrasting the effects we observed when disrupting the entire eIF3 complex or targeting its mRNA-entry-channel arm disentangles the contributions of the eIF3i and eIF3g subunits from those of the other subunits of the complex. These analyses provide evidence that eIF3 and its mRNA-entry-channel arm collaborate functionally with eIF4A, eIF4B, and Ded1 to drive initiation on mRNAs with long $5^{\prime}$-UTRs and with a lower propensity to form stable closed-loop structures mediated by eIF4G, eIF4E, and PABP. They further reveal that eIF3 stimulates the translation of mRNAs involved in mitochondrial processes and contributes to the resolution of structurally complex regions during initial docking or scanning, and that these roles require subunits beyond eIF3i and eIF3g.

\section{MATERIALS AND METHODS}

\section{Cell Growth and Harvest}

We created ribosome profiling libraries from eIF3 mutant and corresponding isogenic WT strains for both the eIF3a/b Degron (YAJ34: MATa trp1D leu2-3,112 ura3-52 gen2::hisG $P_{\text {GAL1 }}$-mycUBR1::TRP1::ubr1 $P_{C U P 1^{-}}$UBI-R-HA-tif32 ${ }^{t d}:: U R A 3:: t i f 32 P_{C U P 1}-U B I-$ $R-D H F R^{t s}-H A-p r t 1^{t d}:: U R A 3:: p r t 1$ and YAJ3: MATa trp1Dleu23,112 ura3-52 gcn2::hisG $P_{\text {GAL1-myc-UBR1::TRP1::ubr1 pRS316 }}$ [URA3]) (Jivotovskaya et al., 2006) and eIF3i DDKK (H450: MATa leu2-3,-112 ura3-52::GCN2 trp1s tif34A hc TIF34 URA3 transformed with YCp-i/TIF34-D207K-D224K-HA or YCp-i/ TIF34-HA, respectively) (Herrmannová et al., 2012) as described previously (Ingolia, 2010; Sen et al., 2015; Sen et al., 2016). We grew two biological replicates of each strain and its matching isogenic WT strain under permissive conditions before harvesting and adding to pre-warmed restrictive media for a duration resulting in an $\sim 90 \%$ decrease in bulk translation (as judged by polysome:monosome ratios, Supplementary Figure S1) and a final cell density at midlog phase $\left(\mathrm{OD}_{600}=\sim 0.6\right)$. We grew eIF3a/b Degron cells at $25^{\circ} \mathrm{C}$ in $\mathrm{SC}_{\mathrm{Raff}}+\mathrm{Cu}^{2+}$ before shifting them to pre-warmed $\mathrm{SC}_{\mathrm{Raff} / \mathrm{Gal}}+\mathrm{BCS}$ at $36^{\circ} \mathrm{C}$ for $90 \mathrm{~min}$. We grew eIF3i DDKK at $30^{\circ} \mathrm{C}$ in SC media before shifting them to pre-warmed SC media at $37^{\circ} \mathrm{C}$ for $30 \mathrm{~min}$. We added cycloheximide to a final concentration of $100 \mu \mathrm{g} / \mathrm{ml} 2 \mathrm{~min}$ prior to harvesting by filtration through a Kontes filtration apparatus and flash freezing in liquid nitrogen with $2 \mathrm{ml}$ of ribosome footprinting buffer (20 mM Tris $\mathrm{pH} 8.0,140 \mathrm{mM} \mathrm{KCl}, 1.5 \mathrm{mM} \mathrm{MgCl} 2,1 \%$ Triton, $100 \mu \mathrm{g} / \mathrm{ml}$ cycloheximide).

\section{Ribosome Profiling and RNA-Seq Library Preparation}

We generated sequencing libraries of ribosome footprints and total mRNA as previously described (Ingolia, 2010; Sen et al.,
2015; Sen et al., 2016). Briefly, we lysed cells using a freezer mill and then prepared lysates by centrifuging $5 \mathrm{~min}$ at $3,000 \times \mathrm{g}$, collecting the supernatant and then centrifuging $12 \mathrm{~min}$ at $>20,000 \times$ g. We then collected the supernatant and flashfroze in $30 \mathrm{OD}_{260}$ aliquots. We purified ribosome footprints by adding $5 \mu \mathrm{L}$ RNasel to one aliquot of purified lysate and incubating $60 \mathrm{~min}$ at $26^{\circ} \mathrm{C}$ with mixing at $700 \mathrm{rpm}$. We then added $5 \mu \mathrm{L}$ SuperAsin (Thermo Fisher) and loaded on a $10-50 \%$ sucrose gradient and centrifuged at 40,000 rpm for $3 \mathrm{~h}$ and then collected the monosome peak using a gradient fractionator. We then purified RNA from purified monosomes via hot phenol extraction. We purified total mRNA from one $30 \mathrm{OD}_{260}$ aliquot of purified lysate using the miRNeasy mini kit (Qiagen) per the vendor's instructions and then randomly fragmented at $70^{\circ} \mathrm{C}$ for 8 min using Fragmentation Reagent (Invitrogen). We then performed subsequent steps (size selection, linker ligation, reverse transcription, circularization, rRNA subtraction, and PCR amplification) as previously described and had libraries sequenced using an Illumina HiSeq system.

\section{Analysis of Sequencing Data}

We processed and analyzed sequencing libraries of ribosome footprints and total mRNA as described previously (Ingolia, 2010; Sen et al., 2015; Sen et al., 2016). We then employed DESeq2 (Love et al., 2014) for statistical analysis of differences in ribosome footprint and RNA-seq read counts, and $\mathrm{TE}_{\text {rel }}$ values between WT and mutant samples, as previously reported (Martin-Marcos et al., 2017; Kulkarni et al., 2019). We excluded genes with fewer than 128 total mRNA reads in the four samples combined (two replicates of both WT and mutant strains) from the calculation of $\mathrm{TE}_{\text {rel }}$ values. We then performed subsequent analysis of mRNA features and characteristics within $\mathrm{R}$, using custom scripts, together with previously-reported datasets reporting $5^{\prime}$-UTR lengths, PARS values, closed-loopforming propensity of individual mRNAs, previously identified $\mathrm{uORFs}$, or $\triangle \mathrm{TE}_{\text {rel }}$ values observed in the presence of mutations targeting eIF4A, eIF4B, Ded1, eIF1, or eIF1A. We performed Gene Ontology analysis using the Gene Ontology Resource PANTHER classification system. Statistical tests were performed as described in the main text and figures.

\section{RESULTS}

\section{Disruption of the elF3 Complex Provokes Severe Translational Defects}

To investigate the transcriptome-wide roles of eIF3, we performed ribosome profiling in two $S$. cerevisiae strains in which the eIF3 complex is partially or entirely compromised. The eIF3a/b Degron strain expresses temperature-sensitive degron variants of the eIF3a and eIF3b subunits. Depletion of these subunits provokes the loss of the entire eIF3 complex (Jivotovskaya et al., 2006). The eIF3i DDKK strain expresses a variant of the eIF3i subunit that is unable to bind stably to eIF3b (Herrmannová et al., 2012). Because both eIF3i and eIF3g depend on this interaction to associate with the remainder of the eIF3 complex, this results in the loss of both subunits, which normally 
A

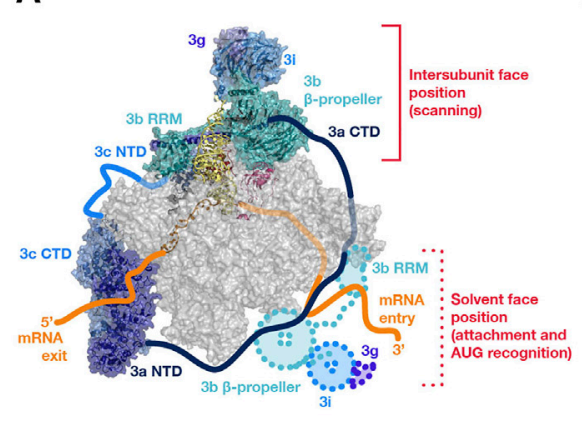

C

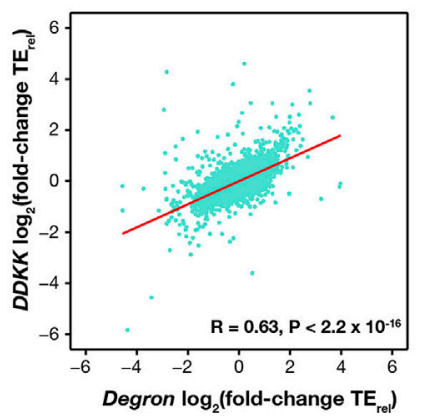

B

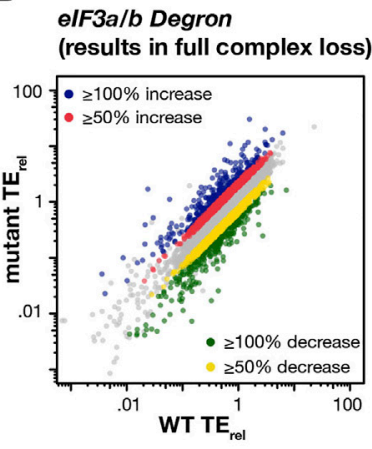

D

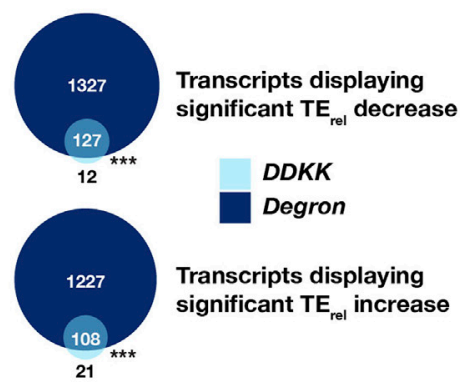

elF3i DDKK

(results in loss of $\mathrm{i}$ and $\mathrm{g}$ )

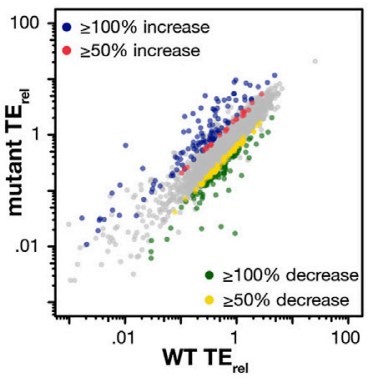

E

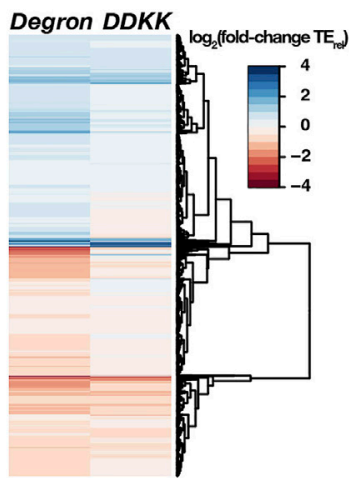

FIGURE 1 | Disruption of the elF3 complex or its mRNA-entry channel arm provoke strong and overlapping effects on the translation of mRNAs across the transcriptome. (A) Structural model of elF3 bound to the PIC (PDB 6GSM) viewed looking down at top of the small (40S) ribosomal subunit head so as to visualize the path of mRNA as it enters and exits the PIC (Llácer et al., 2021). The small (40S) ribosomal subunit is shown in grey, and the initiator tRNA and mRNA are shown in yellow and orange, respectively (with the path of the mRNA entering and exiting the PIC shown as a cartoon). Subunits of the elF3 complex are shown in blues and greens, with the mRNA-entry-channel arm shown in two positions: at the intersubunit face of the PIC (identified density seen in this structure) or at the solvent face (cartoons depicting approximate location observed in structures lacking mRNA or after start-codon recognition). (B) $\mathrm{TE}_{\text {rel }}$ correlation plots comparing observed $\mathrm{TE}_{\text {rel }}$ values in either elF3a/b Degron (left) or elF3i DDKK (right) cells with TE $\mathrm{E}_{\text {rel }}$ values observed in isogenic wild-type cells. Transcripts displaying significant $\left(P_{\text {adj }}<0.05\right) T E_{\text {rel }}$ increases ( $\geq 50 \%$ red and $\geq 100 \%$ blue) or decreases $(\geq 50 \%$ yellow and $\geq 100 \%$ green) as determined by DESeq2 analysis are shown in color. (C) Correlation plot comparing observed TE $\mathrm{E}_{\mathrm{rel}}$ changes in elF3a/b Degron and elF3i DDKK cells, with predicted Pearson correlation shown in red. (D) Venn diagrams describing the overlap in transcripts displaying significant $\mathrm{TE}_{\text {rel }}$ decreases (top) or increases (bottom) in elF3a/b Degron (dark blue) or elF3i DDKK (light blue) cells. ${ }^{\star \star \star}=\mathrm{P}<10^{-10},{ }^{\star \star}=\mathrm{P}<10^{-5},{ }^{\star}=\mathrm{P}<0.05$, HyperGeometric test. (E) Heatmap and dendrogram resulting from hierarchical clustering analysis of significant $\mathrm{TE}_{\mathrm{rel}}$ changes observed in elF3a/b Degron and elF3i $D D K K$ cells.

contribute to the mRNA-entry-channel arm of eIF3 (Figure 1A). This arm has been observed in two distinct locations of the PIC, depending on its functional state (Llácer et al., 2015; Llácer et al., 2018).

Before constructing ribosome profiling libraries, we first investigated the effects of the eIF3a/b Degron and eIF3i DDKK mutations on global translation levels, as assayed by polysome profiling. For each strain, we grew cells first under permissive conditions and then shifted them to restrictive conditions for 30 , 60,90 , and $120 \mathrm{~min}$. Both strains exhibit no growth defect under permissive conditions but manifest severe growth defects at restrictive conditions (Jivotovskaya et al., 2006; Herrmannová et al., 2012). Consistent with this, polysome profiles collected under permissive growth conditions for both strains were similar to those collected for isogenic wild-type strains (Supplementary Figure S1A). In contrast, we observed strong decreases in polysome to monosome ratios $(\mathrm{P} / \mathrm{M})$ upon shifting to restrictive conditions for both strains, with $e I F 3 a / b$ Degron and eIF3i DDKK cells exhibiting an approximately $90 \%$ decrease in $\mathrm{P} / \mathrm{M}$ (as compared to isogenic WT cells grown under the same conditions) after $30 \mathrm{~min}$ and $90 \mathrm{~min}$ at restrictive conditions, respectively (Supplementary Figure S1B,C).

\section{Relative TE Changes Identify mRNAs Most or Least Sensitive to the Disruption of the Entire elF3 Complex or Its mRNA-Entry- Channel Arm}

Given the marked decrease in global translation levels we observed in both strains, we next asked how these global affects translate to individual mRNAs across the transcriptome. To that end, we constructed ribosome profiling and RNA-seq libraries from both the eIF3i DDKK and $e I F 3 a / b$ Degron strains (and their corresponding isogenic WT strains) grown under restrictive conditions and calculated relative translational efficiency $\left(\mathrm{TE}_{\mathrm{rel}}\right)$ values for coding sequences (CDS), ignoring reads obtained from the initial 15 codons and 
from the final 5 codons to avoid cycloheximide-induced artifacts (Gerashchenko and Gladyshev, 2014). Owing to the absence of an internal read-count standard, read counts from both ribosome profiling and RNA-seq libraries are normalized to the total library size for each condition. The $\mathrm{TE}_{\mathrm{rel}}$ values we calculate from these normalized read counts thus do not enable direct comparison of absolute $\mathrm{TE}$ between samples. Instead, $\mathrm{TE}_{\text {rel }}$ values provide a measure of the translational status of individual mRNAs as compared to the overall population of mRNAs within each sample. We also calculated $\mathrm{TE}_{\text {rel }}$ values for a set of previously identified uORFs (Martin-Marcos et al., 2017; Kulkarni et al., 2019) but did not attempt to identify novel translated uORFS owing to the inclusion of cycloheximide in our library preparation. Both ribosome footprint and RNA-seq libraries were highly reproducible across replicates for each condition (Supplementary Figure S2).

Using the R DESeq2 package (Love et al., 2014), we then identified transcripts whose $\mathrm{TE}_{\text {rel }}$ was significantly changed $\left(\mathrm{P}_{\mathrm{adj}}<0.05\right)$ in each strain, as compared to an isogenic WT strain (Figure 1B). Owing to the marked decrease in global translational levels we observed in each mutant strain, as well as the normalization to total ribosomal footprint reads performed when calculating $\mathrm{TE}_{\text {rel }}$, we interpreted mRNAs exhibiting significant $\mathrm{TE}_{\text {rel }}$ decreases as having a greater than average dependence on the either the eIF3i and eIF3g subunits lost in eIF3i DDKK cells or on the entire eIF3 complex disrupted in $e I F 3 a / b$ Degron cells. We interpreted those mRNAs exhibiting significant $\mathrm{TE}_{\mathrm{rel}}$ increases as instead having a weaker than average dependance on the regions of the eIF3 complex targeted by each mutation. The significant effects on global translational levels that we observed in both eIF3i DDKK and eIF3a/b Degron cells in fact suggest that most mRNAs likely experience decreases in their absolute TE. Nonetheless, comparison of these significant changes in $\mathrm{TE}_{\mathrm{rel}}\left(\Delta \mathrm{TE}_{\mathrm{rel}}\right)$ enables identification of those mRNAs whose translation is most or least sensitive to disruption of eIF3 or its mRNA-entry-channel arm in a background where global translational levels are repressed.

In $e I F 3 a / b$ Degron cells-in which the entire eIF3 complex is disrupted-we identified 1,455 transcripts whose $\mathrm{TE}_{\mathrm{rel}}$ decreased and 1,340 transcripts whose $\mathrm{TE}_{\mathrm{rel}}$ increased $(5,466$ total with significant read counts), as compared to $\mathrm{TE}_{\text {rel }}$ values in an isogenic WT strain (Figure 1B). Because eIF3 has been implicated in mediating the translation of specific mRNAs in a number of cell types (Sha et al., 2009; Lee et al., 2015; Rode et al., 2018; Lin et al., 2020; Lamper et al., 2020), we investigated the gene ontology (GO) terms associated with these affected mRNAs. The set of transcripts whose $\mathrm{TE}_{\text {rel }}$ decreased in eIF3a/b Degron cells was enriched for mRNAs with GO terms involved in mitochondrial translation and gene expression, as well as a variety of metabolic processes (Supplementary Figure S3). These most sensitive mRNAs were under-enriched in mRNAs involved in RNA processing and ribosome biogenesis. Consistent with this, mRNAs whose $\mathrm{TE}_{\mathrm{rel}}$ increased in $e I F 3 a / b$ Degron cells were enriched in GO terms associated with RNA processing and under-enriched in terms associated with mitochondrial translation and gene expression (Supplementary Figure S3).

In eIF3i DDKK cells-in which the eIF3i and eIF3g subunits of the eIF3 mRNA-entry-channel arm are lost from the complex-we identified 139 transcripts whose $\mathrm{TE}_{\text {rel }}$ decreased and 133 transcripts whose $\mathrm{TE}_{\text {rel }}$ increased, as compared to $\mathrm{TE}_{\mathrm{rel}}$ values in an isogenic WT strain (Figure 1B). We did not observe any significant over- or under-enrichment of specific GO terms in affected transcripts in these cells, perhaps because the strong global effects on translation in eIF3i DDKK cells are more uniformly distributed amongst all mRNAs, resulting in widespread but uniform decreases in absolute TE levels across the transcriptome with more limited effects on the relative TE of individual mRNAs.

In both eIF3i DDKK and eIF3a/b Degron cells, we observed strong increases in uORF translation (Supplementary Figure S4A). Of 4,830 uORFs identified previously, 529 displayed significant $\left(\mathrm{P}_{\mathrm{adj}}<0.05\right)$ increases in $\mathrm{TE}_{\mathrm{rel}}$ in eIF3a/b Degron cells, whereas 21 displayed significant decreases in $\mathrm{TE}_{\mathrm{rel}}$. In $e I F 3 i$ DDKK cells, 391 uORFs displayed significant $\left(\mathrm{P}_{\text {adj }}<0.05\right)$ increases in $\mathrm{TE}_{\text {rel }}$, whereas 5 displayed significant decreases in $\mathrm{TE}_{\text {rel. }}$. This global increase in uORF translation in both strains is likely a result of the previously described effects of cycloheximide on read counts near the start codon (Gerashchenko and Gladyshev, 2014), which cannot be discarded as in the calculation of ORF $\mathrm{TE}_{\text {rel }}$ values owing to the short length of these regions. Thus we focused subsequent analysis of $\mathrm{uORF} \mathrm{TE}_{\mathrm{rel}}$ values on any observed differential behavior between uORFs within the same strain.

Because the eIF3 complex is either partly or entirely disrupted in both cell lines, we investigated the degree to which the $\mathrm{TE}_{\mathrm{rel}}$ effects we observed were similar in eIF3i DDKK and eIF3a/b Degron cells. We observed a significant correlation $(\mathrm{R}=0.63, \mathrm{P}<$ $2.2 \times 10^{-16}$ ) between the $\Delta \mathrm{TE}_{\text {rel }}$ values observed in both strains (Figure 1C). Additionally, we found a strong overlap between those transcripts exhibiting $\mathrm{TE}_{\text {rel }}$ decreases in eIF3i DDKK and $e I F 3 a / b$ Degron cells (Figure 1D), with the changes observed in eIF3i DDKK cells appearing to represent a subset of the changes observed in $e I F 3 a / b$ Degron cells. Moreover, the overall portfolio of $\Delta \mathrm{TE}_{\mathrm{rel}}$ values we observe is similar for both strains, as evidenced by heat map comparison of the magnitude and direction of observed changes (Figure 1E). Together, these results are consistent with strong translational effects upon disruption of the eIF3 mRNA-entry-channel arm (as in eIF3i $D D K K$ cells) or depletion of the entire eIF3 complex (as in $e I F 3 a / b$ Degron cells) and a role for eIF3 in promoting the translation of mRNAs with mitochondrial roles.

\section{mRNAs Most Sensitive to the elF3a/b Degron or elF3i DDKK Mutations Possess Longer 5'-Untranslated Regions}

We next investigated the structural features (Figure 2A) of mRNAs most or least sensitive to the eIF3i DDKK and eIF3a/ $b$ Degron mutations, as identified by DESeq2 analysis of $\mathrm{TE}_{\mathrm{rel}}$ changes in each strain. eIF3 is required for the overall process of mRNA recruitment both in vivo (Jivotovskaya et al., 2006) and 


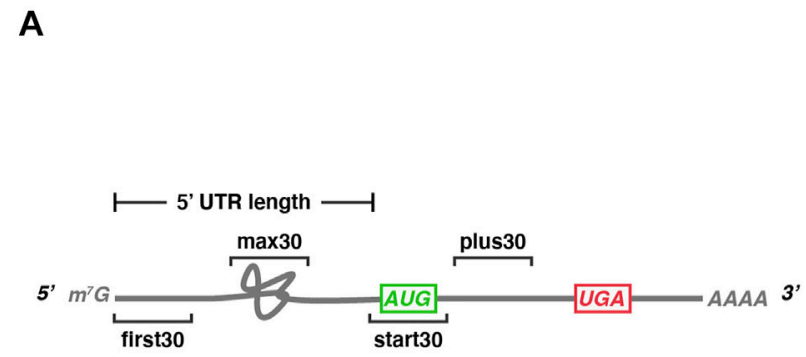

C 追 $+\Delta \mathrm{TE}_{\mathrm{rel}}$ 白 unchanged 白 $-\Delta \mathrm{TE}_{\mathrm{rel}}$
B

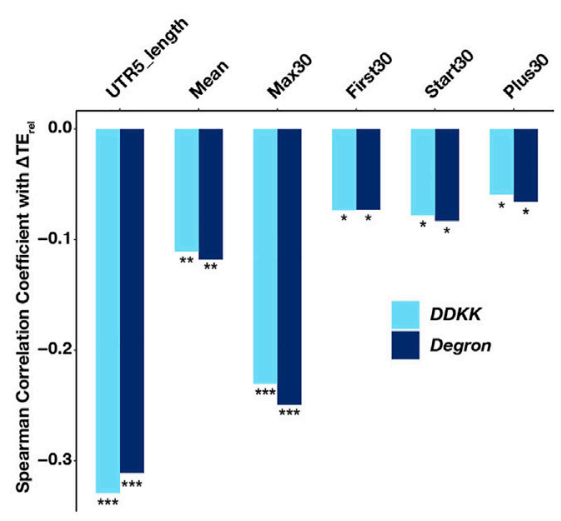

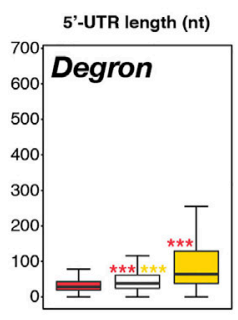
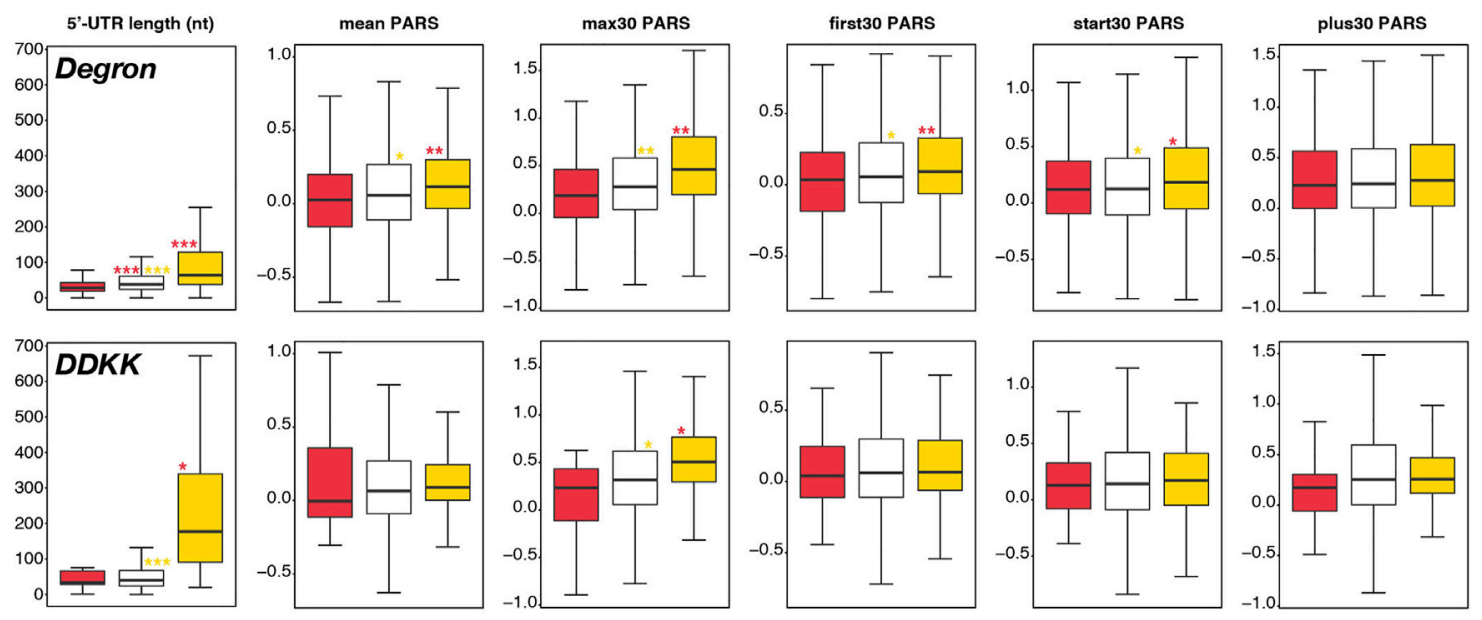

FIGURE 2 | Transcripts most sensitive to disruption of the elF3 complex or its mRNA-entry-channel arm possess longer 5'-UTRs. (A) Cartoon depicting a hypothetical mRNA and detailing the $5^{\prime}$-UTR length and the specific 30-nt windows within which mean PARS values (Kertesz et al., 2010 ) were calculated as measures of structural complexity. (B) Bar plot comparing the Spearman correlation coefficients obtained when comparing observed $\Delta \mathrm{TE}_{\text {rel }}$ values in each mutant elF3 strain and different measures of $5^{\prime}$-UTR length or complexity. ${ }^{\star \star \star}=\mathrm{P}<10^{-10},{ }^{\star \star}=\mathrm{P}<10^{-5},{ }^{\star}=\mathrm{P}<0.05$. (C) Box and whisker plots comparing different measures of $5^{\prime}$-UTR

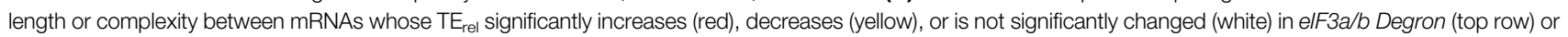
elF3i DDKK (bottom row) cells. ${ }^{\star \star *}=\mathrm{P}_{\mathrm{adj}}<10^{-10}{ }^{\star \star *}=\mathrm{P}_{\mathrm{adj}}<10^{-5},{ }^{\star}=\mathrm{P}_{\mathrm{adj}}<0.05$, color indicates comparison set; Wilcoxon Test for $5^{\prime}$-UTR lengths, ANOVA with post-hoc Tukey test for others.

in vitro (Mitchell et al., 2010; Aitken et al., 2016), and mutations to several eIF3 subunits elicit defects in mRNA recruitment or its component events of initial docking, scanning, and start-codon recognition (Valášek et al., 2002; Nielsen et al., 2004; Chiu et al., 2010; Cuchalova et al., 2010; Elantak et al., 2010; Herrmannová et al., 2012).

To shed light on the contribution of the eIF3 complex and the eIF3i and eIF3g subunits of the mRNA-entry-channel arm to scanning processivity, we first asked if the $\Delta \mathrm{TE}_{\text {rel }}$ values we observed in each strain correlated with $5^{\prime}$-UTR length. Upon restricting our analysis to mRNAs previously shown to have a dominant $5^{\prime}$-UTR isoform (defined as mRNAs for which one isoform accounts for at least $40 \%$ of all transcripts and is present at an abundance at least twice that of the next most abundant isoform) (Pelechano et al., 2013; Zinshteyn et al., 2017), we observed significant $\left(\mathrm{P}<10^{-59}\right)$ negative correlations between $5^{\prime}$-UTR length and $\triangle \mathrm{TE}_{\text {rel }}$ in both the eIF3i DDKK and $e I F 3 a / b$ Degron strains (Figure 2B). Consistent with this effect, we also observed a negative correlation between $\Delta \mathrm{TE}_{\text {rel }}$ and distance from the $5^{\prime}$ end for a set uORFs identified in previous studies (MartinMarcos et al., 2017; Kulkarni et al., 2019), though this correlation was significant only in eIF3i DDKK cells (Supplementary Figure S4B).

To interrogate the roles of eIF3 and the eIF3i and eIF3g subunits in resolving structural impediments during initial mRNA docking or scanning, we next determined the relationship between $\Delta \mathrm{TE}_{\mathrm{rel}}$ and the propensity of specific regions of an mRNA to form secondary structures, as measured by their differential sensitivity in vitro to nucleases specific for single- or double-stranded RNA (PARS, Figure 2A) (Kertesz et al., 2010). In eIF3a/b Degron and eIF3i DDKK cells, we observed a significant $\left(\mathrm{P}<10^{-8}\right.$ and $\mathrm{P}<10^{-7}$, respectively) negative correlation between $\triangle \mathrm{TE}_{\text {rel }}$ and mean $5^{\prime}$-UTR PARS values, as well as with mean PARS scores determined within specific 30 nucleotide (nt) windows located at the first $30 \mathrm{nt}$ of the $5^{\prime}$-UTR (First30; $\mathrm{P}<10^{-3}$ for both), $30 \mathrm{nt}$ centered around the 
AUG start codon (Start30; $\mathrm{P}<10^{-4}$ for both), and the first $30 \mathrm{nt}$ downstream of the Start30 window (Plus30; $\mathrm{P}<10^{-3}$ and $\mathrm{P}<$ $10^{-2}$, respectively) (Figure 2B) (Sen et al., 2015; Sen et al., 2016). Of the correlations we observed between $\triangle \mathrm{TE}_{\mathrm{rel}}$ and PARS measures, the strongest and most significant was with the maximum PARS score observed within any $30 \mathrm{nt}$ window within the $5^{\prime}$-UTR (Max30; $\mathrm{P}<10^{-32}$ and $\mathrm{P}<10^{-38}$, respectively) (Figure 2B).

Having observed these correlations between $\Delta \mathrm{TE}_{\mathrm{rel}}$ and $5^{\prime}$ UTR length or structural complexity in both the eIF3i DDKK and $e I F 3 a / b$ Degron strains, we next investigated if we could detect significant differences in the median values of these measures when comparing mRNAs whose $\mathrm{TE}_{\text {rel }}$ was either significantly decreased or increased in either strain (Figure 2C). As before, we restricted our analysis to mRNAs with one dominant $5^{\prime}$ isoform. In $e I F 3 a / b$ Degron cells, mRNAs displaying significant negative $\Delta \mathrm{TE}_{\text {rel }}$ values possess longer $5^{\prime}$-UTRs and those displaying significant positive $\Delta \mathrm{TE}_{\text {rel }}$ values possess shorter $5^{\prime}$-UTRs, as compared to mRNAs whose $\mathrm{TE}_{\text {rel }}$ was not significantly affected ( $\mathrm{P}_{\mathrm{adj}}<10^{-28}$ for all pairwise comparisons, Wilcoxon test). Similarly, in eIF3i DDKK cells, mRNAs displaying significant negative $\Delta \mathrm{TE}_{\text {rel }}$ values possess longer $5^{\prime}$-UTRs than both unaffected mRNAs $\left(\mathrm{P}_{\mathrm{adj}}<10^{-18}\right)$ and mRNAs displaying significant positive $\Delta \mathrm{TE}_{\text {rel }}$ values $\left(\mathrm{P}_{\mathrm{adj}}<10^{-3}\right)$. However, we did not observe a significant difference in $5^{\prime}$-UTR lengths when comparing mRNAs displaying significant positive $\Delta \mathrm{TE}_{\mathrm{rel}}$ values and unaffected mRNAs in eIF3i DDKK cells, perhaps because the set of mRNAs expressed as a dominant transcript isoform and exhibiting significant positive $\Delta \mathrm{TE}_{\text {rel }}$ values in these cells is relatively small $(n=57)$. In both cell lines, we observed similar results when comparing $5^{\prime}$-UTR values reported in a separate study (Kertesz et al., 2010) (Supplementary Figure S5A).

When comparing measures of structural complexity (as measured by PARS values across the $5^{\prime}$-UTR and in distinct windows), we again observed differences between mRNAs whose $\mathrm{TE}_{\text {rel }}$ either increased or decreased in eIF3a/b Degron cells. mRNAs displaying significant $\mathrm{TE}_{\text {rel }}$ decreases in these cells have higher mean and $\max 305^{\prime}$-UTR PARS values, as compared to unaffected mRNAs $\left(\mathrm{P}_{\mathrm{adj}}<10^{-3}\right.$ and $\mathrm{P}_{\mathrm{adj}}<10^{-6}$, respectively; ANOVA and post-hoc Tukey test) and mRNAs displaying significant $\mathrm{TE}_{\mathrm{rel}}$ increases $\left(\mathrm{P}_{\mathrm{adj}}<10^{-9}\right.$ and $\mathrm{P}_{\mathrm{adj}}<$ $10^{-6}$, respectively). Similarly, mRNAs whose $\mathrm{TE}_{\text {rel }}$ decreased in $e I F 3 a / b$ Degron cells have higher PARS values at the $5^{\prime}$ end of their $5^{\prime}$-UTRs (first30) and around their start codons (start30) as compared to mRNAs whose $\mathrm{TE}_{\mathrm{rel}}$ either increased $\left(\mathrm{P}_{\mathrm{adj}}<10^{-7}\right.$ and $\mathrm{P}_{\text {adj }}<10^{-2}$, respectively) or was unaffected $\left(\mathrm{P}_{\mathrm{adj}}<10^{-2}\right.$ for both) in these cells. However, these differences appear more modest than those observed for $5^{\prime}$-UTR length, mean and max30 PARS values. In contrast, there is no significant difference in the PARS values downstream of the start-codon window (plus30) between mRNAs whose $\mathrm{TE}_{\text {rel }}$ either increased, decreased, or was unaffected in $e I F 3 a / b$ Degron cells.

In eIF3i DDKK cells, we did not observe significant differences in most measures of $5^{\prime}$-UTR structural complexity when comparing mRNAs whose $\mathrm{TE}_{\text {rel }}$ either decreased, increased, or was unaffected. The one exception is the max30 PARS values of

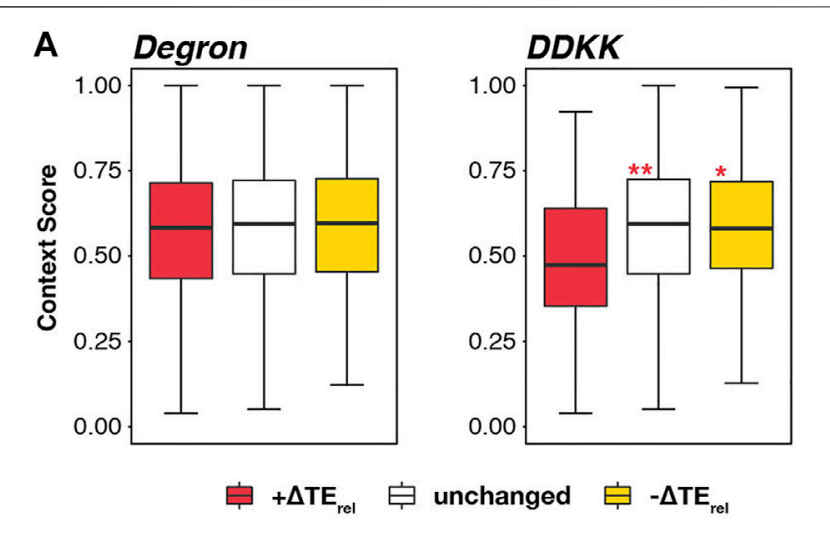

B

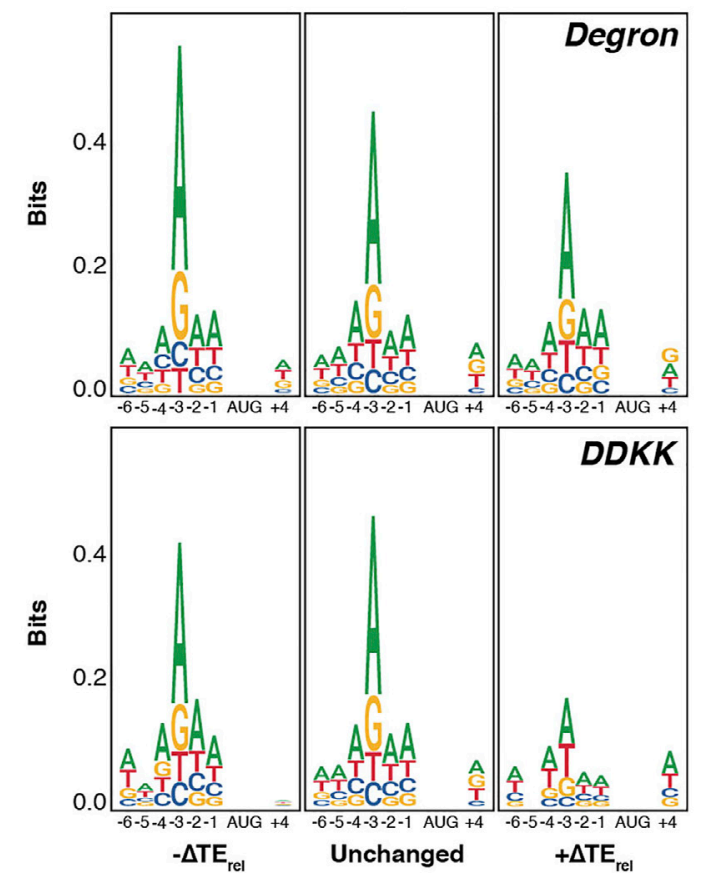

FIGURE 3 | Disruption of the elF3 mRNA-entry-channel arm interferes with discrimination against start codons appearing in poor Kozak sequence context. (A) Box and whisker plots comparing the Kozak sequence context (nt -6 thru +4 ) of transcripts whose $\mathrm{TE}_{\mathrm{rel}}$ significantly increases (red), decreases (yellow), or is not significantly changed (white) in each elF3 mutant strain. ${ }^{\star \star \star}=\mathrm{P}_{\mathrm{adj}}<10^{-10},{ }^{\star \star}=\mathrm{P}_{\mathrm{adj}}<10^{-5},{ }^{*}=\mathrm{P}_{\mathrm{adj}}<0.05$, color indicates comparison set; ANOVA with post-hoc Tukey test. (B) Sequence logos of $\mathrm{nt}-6$ to +4 of transcripts whose $T E_{\text {rel }}$ significantly increases, decreases, or is not significantly changed in each elF3 mutant strain.

mRNAs whose $\mathrm{TE}_{\text {rel }}$ decreased in these cells, which is higher than for mRNAs whose $\mathrm{TE}_{\text {rel }}$ was not significantly affected $\left(\mathrm{P}_{\mathrm{adj}}<\right.$ $10^{-3}$ ) and modestly different than for transcripts whose $\mathrm{TE}_{\text {rel }}$ increased in these cells $\left(\mathrm{P}_{\mathrm{adj}}<0.02\right)$, perhaps because the number of transcripts displaying significant $\mathrm{TE}_{\text {rel }}$ increases in these cells and with available PARS scores is limited $(n=17)$. Nonetheless, these observations are consistent with a role for eIF3 and its mRNA-entry-channel arm in processive scanning through longer $5^{\prime}$-UTRs. eIF3 also appears to contribute to resolving structural complexity within the $5^{\prime}$-UTR during initial docking or scanning, 
though the mRNA-entry-channel arm may play a more peripheral role in these events.

\section{Disruption of the elF3 mRNA-Entry-Channel Arm Exerts Modest Effects on Discrimination Against Start Codons in Poor Kozak Sequence Context}

Because various subunits of eIF3 appear to play roles in startcodon recognition (Nielsen et al., 2004; Valasek et al., 2004; Chiu et al., 2010; Cuchalova et al., 2010; Herrmannová et al., 2012), we next asked whether there was a correlation between the observed $\Delta \mathrm{TE}_{\mathrm{rel}}$ values in the eIF3i DDKK and eIF3a/b Degron strains and the strength of the Kozak consensus sequence surrounding the AUG start codon for each mRNA. However, we did not observe a significant correlation between context scores (calculated for nucleotides -6 to +4$)$ and $\Delta \mathrm{TE}_{\text {rel }}$ values in either eIF3i DDKK or eIF3a/b Degron cells (Supplementary Figure S6).

Consistent with this, we observed no significant difference in the median context scores for mRNAs whose $\mathrm{TE}_{\text {rel }}$ either increased, decreased, or was unaffected in eIF3a/b Degron cells (Figure 3A). Nonetheless, we did observe modest but significant differences between the median context scores of mRNAs displaying $\mathrm{TE}_{\mathrm{rel}}$ increases in eIF3i DDKK cells, which are weaker than either those of mRNAs displaying $\mathrm{TE}_{\text {rel }}$ decreases or those whose $\mathrm{TE}_{\text {rel }}$ was unaffected ( $\mathrm{P}_{\mathrm{adj}}<10^{-4}$ and $\mathrm{P}_{\mathrm{adj}}<10^{-5}$, respectively).

Given these modest effects, we investigated the sequence logos in the vicinity of the start codons of these distinct mRNAs (Figure 3B). Here again, we observed no difference when comparing mRNAs whose $\mathrm{TE}_{\text {rel }}$ either increased, decreased, or was unaffected in eIF3a/b Degron cells. However, consistent with the differences we observed in context scores in eIF3i DDKK cells, mRNAs displaying $\mathrm{TE}_{\text {rel }}$ increases in this background show a weaker preference for an adenine at the $-3 \mathrm{nt}$ than do mRNAs whose $\mathrm{TE}_{\mathrm{rel}}$ is either unaffected or decreases; optimal Kozak consensus sequences contain a purine base (most often adenine) at position -3 and a guanosine at position +4 (Hinnebusch, 2014). Together, these results suggest a peripheral role for the eIF3i and eIF3g subunits of the mRNA-entry-channel arm in discriminating against start codons in poor Kozak context.

\section{The Transcriptome-Wide Effects of the elF3a/b Degron and elF3i DDKK Mutations Most Closely Resemble Those Observed for Mutations Targeting Factors Involved in mRNA Recruitment}

In light of previous work implicating eIF3 in both mRNA recruitment and start-codon recognition and our results here, we next compared the effects we observed in $e I F 3 a / b$ Degron and eIF3i DDKK cells to those previously reported for mutations targeting other initiation factors. The effects of mutations targeting eIFA, eIF4B, and Ded1-which appear to contribute either to initial PIC docking or scanning-have previously been investigated using ribosome profiling (Sen et al., 2015, 2016). Specifically, these studies investigated the effect of temperaturesensitive alleles of eIF4A and Ded1, and a deletion of eIF4B.
Importantly, the sequencing libraries from which these datasets were obtained were prepared by addition of cycloheximide to media in which cells exhibited strong translational defects, as were our sequencing libraries.

We found significant overlaps (Figure $\mathbf{4 A}$, left panel) in the specific mRNAs experiencing significant $\mathrm{TE}_{\text {rel }}$ decreases in eIF3i $D D K K$ or eIF3a/b Degron cells and those mRNAs whose $\mathrm{TE}_{\text {rel }}$ was significantly decreased in the presence of mutations targeting eIF4A ( $\mathrm{P}<10^{-6}$ and $\mathrm{P}<10^{-19}$ for eIF3i DDKK and eIF3a/b Degron, respectively; HyperGeometric test), eIF4B ( $\mathrm{P}<10^{-20}$ for both), and Ded1 $\left(\mathrm{P}<10^{-20}\right.$ and $\left.\mathrm{P}<10^{-11}\right)$. We similarly found significant overlaps when comparing the sets of mRNAs whose $\mathrm{TE}_{\text {rel }}$ increased in the presence of these mutations (eIF4A, $\mathrm{P}<10^{-20}$ and $\mathrm{P}<10^{-17}$; eIF4B, $\mathrm{P}<10^{-13}$ and $\mathrm{P}<10^{-20}$; Ded1, $\mathrm{P}<10^{-10}$ and $\mathrm{P}<10^{-11}$ ). Consistent with these overlapping effects, we also observed significant positive correlations between the $\Delta \mathrm{TE}_{\text {rel }}$ values we observed in both eIF3i DDKK and eIF3a/b Degron cells and $\triangle \mathrm{TE}_{\mathrm{rel}}$ values observed in the presence of mutations targeting eIF4A, eIF4B, and Ded1 (Figure 4B and Supplementary Figure S7). Global comparison of the magnitude and direction of observed $\Delta \mathrm{TE}_{\text {rel }}$ values from these distinct experiments further revealed a similar transcriptome-level portfolio of effects (Figure 4C, right panel).

Having observed these similarities, we next compared the effects observed in eIF3i DDKK and eIF3a/b Degron cells to those obtained from cells expressing an eIF1 variant (L96P) that increases recognition of both AUG start codons in poor context and nearcognate uORF start codons (Zhou et al., 2020). The sequencing libraries giving rise to this dataset were also prepared under conditions similar to those we employed in our experiments. In contrast to the significant overlaps we observed when comparing to datasets from eIF4A, eIF4B, or Ded1 mutant cells, we only found a significant overlap between mRNAs displaying $\mathrm{TE}_{\text {rel }}$ decreases in eIF3i DDKK and L96P eIF1 cells $\left(\mathrm{P}<10^{-5}\right)$ and not in eIF3a/b Degron cells or for mRNAs displaying $\mathrm{TE}_{\text {rel }}$ increases in either eIF3 background (Figure 4A). Consistent with this, we observed a significant but negative correlation between $\Delta \mathrm{TE}_{\text {rel }}$ values from eIF3a/b Degron and L96P eIF1 cells and no significant correlation between eIF3i DDKK and L96P eIF1 cells (Figure 4B and Supplementary Figure S7). Moreover, global comparison of the observed $\Delta \mathrm{TE}_{\text {rel }}$ values from these datasets further revealed a distinct pattern of transcriptome-wide effects in L96P eIF1 cells when compared to either eIF3i DDKK or eIF3a/b Degron cells (Figure 4C, left panel). We also observed weak but modestly significant correlations in $\Delta \mathrm{TE}_{\text {rel }}$ values when comparing our eIF3 datasets to a ribosome profiling dataset obtained from cells expressing $R 13 P$ eIF1A, in which discrimination against near-cognate codons or AUG codons in poor context was increased (Supplementary Figure S7). However, the sequencing libraries for this dataset were prepared from cells harvested in the absence of cycloheximide, which complicates their comparison to our sequencing results.

Together with the more pronounced overlap we observed when comparing our datasets with those obtained from cells expressing mutant versions of eIF4A, eIF4B, and Ded1, these results suggest that eIF3 and its mRNA-entry-channel arm contribute to initial docking and scanning of the mRNA, whereas their contributions to start-codon recognition may be less critical or peripheral to those of eIF1 and eIF1A. 


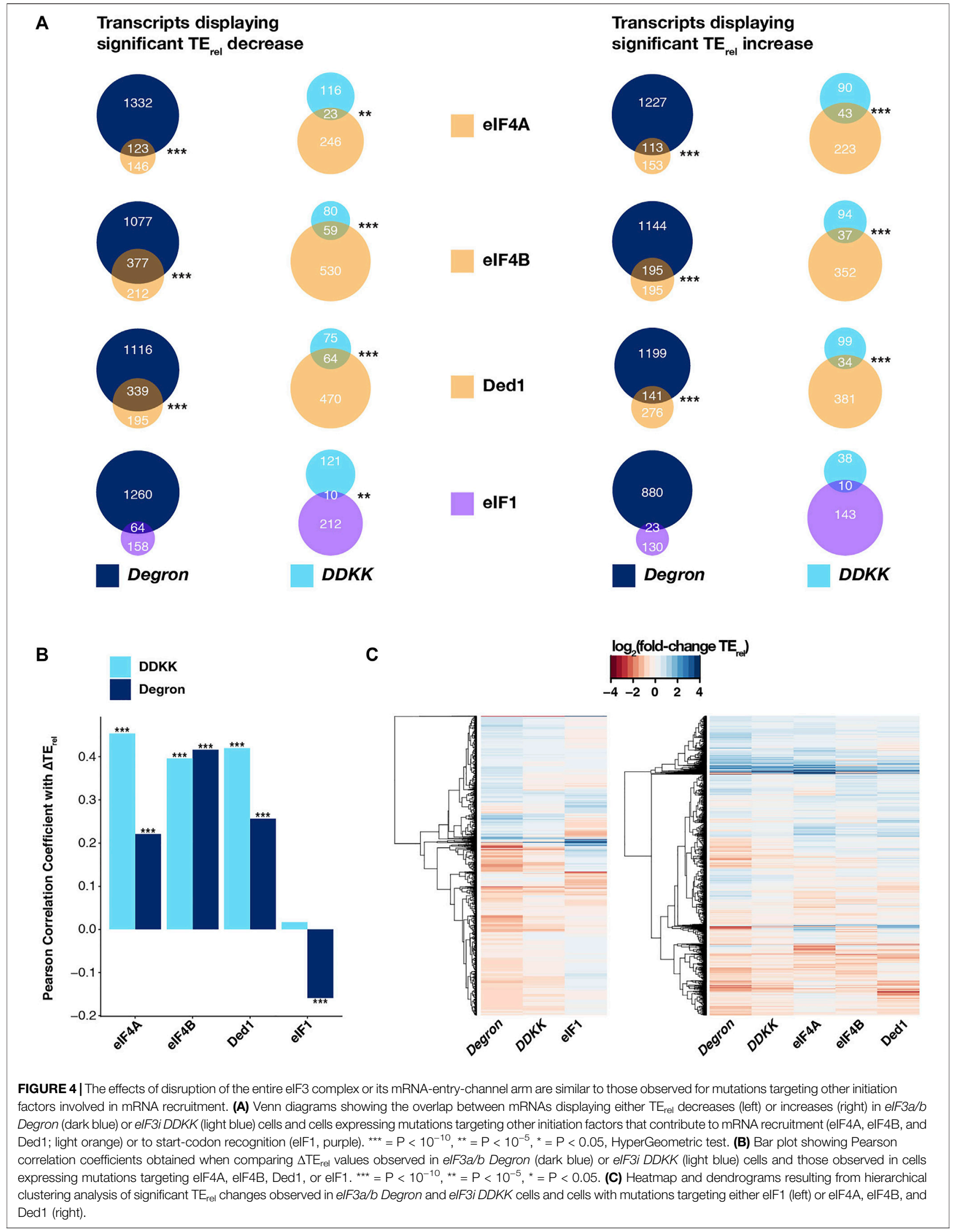




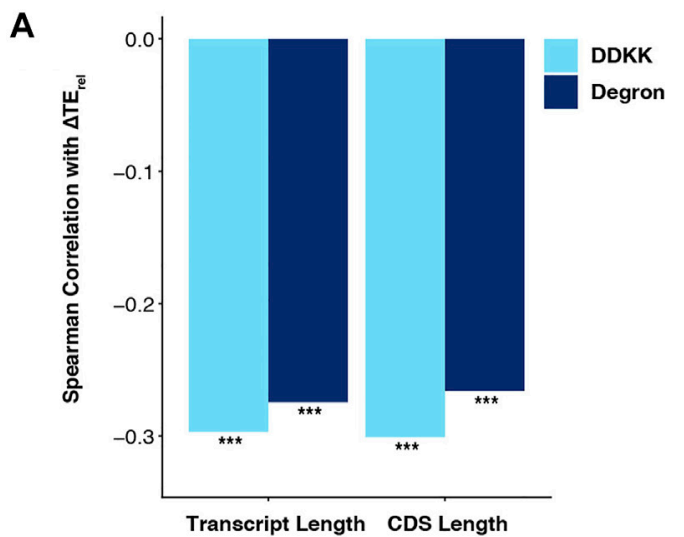

B
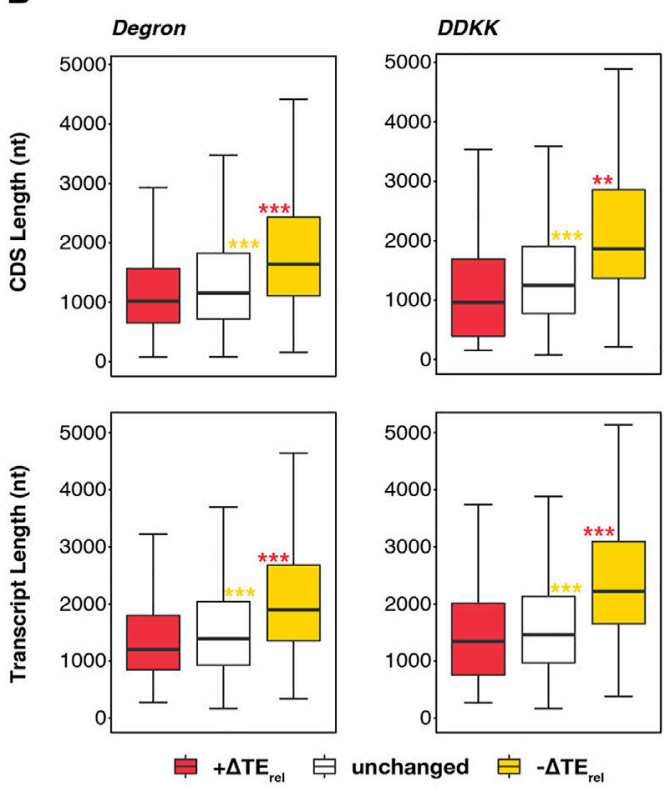

C

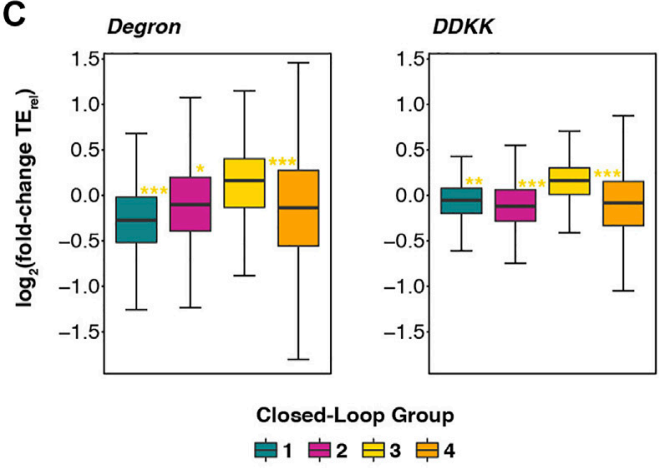

FIGURE 5 | Disruption of the elF3 complex or its mRNA-entrychannel arm most strongly affect long mRNAs with a weaker dependence on closed loop formation. (A) Bar plot showing Spearman correlation coefficients obtained when comparing $\Delta \mathrm{TE}_{\text {rel }}$ values observed in elF3a/b Degron (dark blue) or elF3i DDKK (light blue) cells with overall transcript length and CDS length ${ }^{\star \star *}=\mathrm{P}<10^{-10}{ }^{\star \star *}=\mathrm{P}<10^{-5},{ }^{*}=\mathrm{P}<$

(Continued)
FIGURE 5 | 0.05. (B) Box and whisker plots comparing the CDS and overall transcript length of mRNAs whose $T_{\text {rel }}$ significantly increases (red), decreases (yellow), or is unaffected (white) in either elF3a/b Degron or elF3i DDKK cells. ${ }^{* \star *}=\mathrm{P}_{\mathrm{adj}}<10^{-10},{ }^{* \star}=\mathrm{P}_{\mathrm{adj}}<10^{-5},{ }^{*}=\mathrm{P}_{\mathrm{adj}}<0.05$, color indicates comparison set; Wilcoxon test. (C) Box and whisker plots comparing the $\mathrm{TE}_{\mathrm{rel}}$ changes observed in either elF3a/b Degron (left) or elF3i DDKK (right) cells for previously identified groups of transcripts (Costello et al., 2015) that differentially associate with closed-loop factors such as elF4G, elF4E, and PABP. ${ }^{* \star *}=\mathrm{P}_{\text {adj }}<10^{-10},{ }^{* \star}=\mathrm{P}_{\text {adj }}<10^{-5},{ }^{*}=\mathrm{P}_{\text {adj }}$ $<0.05$, color indicates comparison set; ANOVA and post-hoc Tukey test.

\section{Long Transcripts Less Likely to Form Closed Loop Structures Are More Sensitive to Disruption of the Entire elF3 Complex or Its mRNA-Entry-Channel Arm}

Because of the similarities we observed in the effects of eIF3i $D D K K$ and $e I F 3 a / b$ Degron mutations and mutations targeting eIF4A, eIF4B, and Ded1, we investigated whether these similarities extended to the observation that long transcripts and transcripts with lower closed-loop-forming potential are particularly sensitive to deletion of eIF4B or to mutations of eIF4A or Ded1 (Sen et al., 2016). In fact, we observed significant negative correlations between $\Delta \mathrm{TE}_{\mathrm{rel}}$ and both overall transcript length and coding sequence (CDS) length in both eIF3i DDKK and eIF3a/b Degron cells (Figure 5A). Comparing the overall length of mRNAs whose $\mathrm{TE}_{\mathrm{rel}}$ decreased in each mutant eIF3 background reveals them to be significantly longer than both unaffected mRNAs $\left(\mathrm{P}_{\mathrm{adj}}<10^{-13}\right.$ and $\mathrm{P}_{\mathrm{adj}}<10^{-16}$ for eIF3i DDKK and eIF3a/b Degron, respectively; Wilcoxon test) and mRNAs whose $\mathrm{TE}_{\mathrm{rel}}$ increased $\left(\mathrm{P}_{\mathrm{adj}}<10^{-14}\right.$ and $\left.\mathrm{P}_{\mathrm{adj}}<10^{-16}\right)$ (Figure 5B). Similarly, the CDS lengths of mRNAs whose $\mathrm{TE}_{\text {rel }}$ decreased in the presence of each eIF3 mutation is longer than both unaffected mRNAs $\left(\mathrm{P}_{\mathrm{adj}}<10^{-11}\right.$ and $\left.\mathrm{P}_{\mathrm{adj}}<10^{-16}\right)$ and mRNAs whose $\mathrm{TE}_{\mathrm{rel}}$ increased $\left(\mathrm{P}_{\mathrm{adj}}<10^{-5}\right.$ and $\left.\mathrm{P}_{\mathrm{adj}}<10^{-16}\right)$.

We further observed that, as in cells expressing mutations of eIF4A or Ded1 or in which the gene coding for eIF4B was deleted, mRNAs identified in a previous study (Costello et al., 2015) as having strong closed-loop-forming potential owing to their enrichment in eIF4G, eIF4E, and PABP (group 3) are less sensitive than other mRNAs to both the eIF3i DDKK $\left(\mathrm{P}_{\mathrm{adj}}<10^{-7}\right.$; $\mathrm{P}_{\mathrm{adj}}<10^{-14}$; and $\mathrm{P}_{\mathrm{adj}}<10^{-14}$ for comparison to groups 1,2 , and 4 respectively) or eIF3a/b Degron $\left(\mathrm{P}_{\mathrm{adj}}<10^{-12} ; \mathrm{P}_{\mathrm{adj}}<10^{-4}\right.$; and $\mathrm{P}_{\text {adj }}$ $<10^{-15}$ ) mutations (Figure 5C). Moreover, we observed positive median $\Delta \mathrm{TE}_{\text {rel }}$ values for strong closed-loop mRNAs (group 3) and negative median $\Delta \mathrm{TE}_{\text {rel }}$ values for weak closed-loop mRNAs (groups 1 and 2), suggesting that mRNAs with strong closedloop-forming potential compete more effectively for the initiation machinery when eIF3 or its mRNA-entry-channel arm are disrupted, whereas mRNAs less likely to form closedloop structures are disadvantaged under these conditions. Taken together, these results suggest that, like eIF4A, eIF4B, and Ded1, eIF3 and its mRNA-entry-channel arm may contribute to driving initiation on long mRNAs less likely to form eIF4G-, eIF4E-, and PABP-dependent closed loop structures in vivo. While the translation of most mRNAs likely depends on contributions from these factors, translation of these long and 
$\begin{array}{lll}\text { 早 }+\Delta \mathrm{TE}_{\text {rel }} \text { both } & \text { 帛 }-\Delta \mathrm{TE}_{\text {rel }} \text { both } \\ & +\Delta \mathrm{TE}_{\text {rel }} \text { Degron only } & \text { 帛 }-\Delta \mathrm{TE}_{\text {rel }} \text { Degron only }\end{array}$
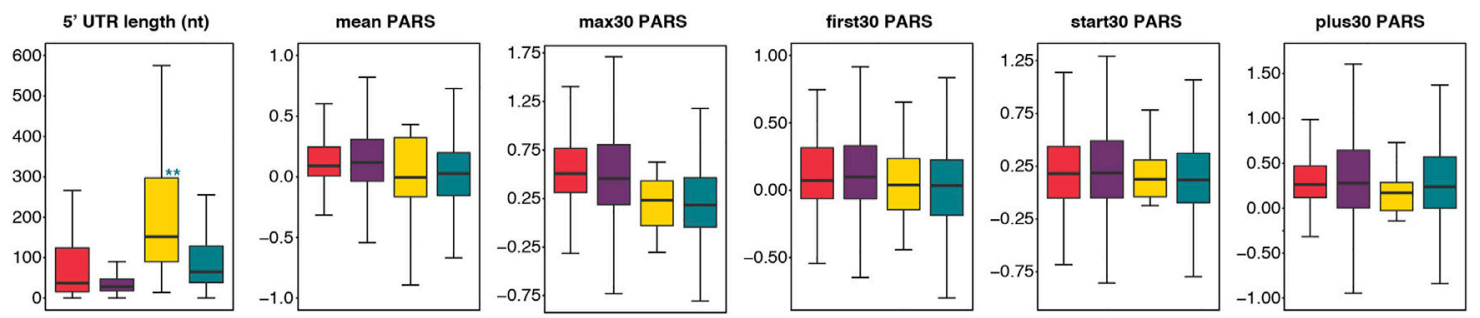

B

$\begin{array}{ll}\text { 早 }+\Delta \mathrm{TE}_{\mathrm{rel}} \text { both } & \text { 泉 }-\Delta \mathrm{TE}_{\mathrm{rel}} \text { both } \\ \text { 帛 }+\Delta \mathrm{TE}_{\mathrm{rel}} \text { Degron only } & \text { 帛 }-\Delta \mathrm{TE}_{\mathrm{rel}} \text { Degron only }\end{array}$
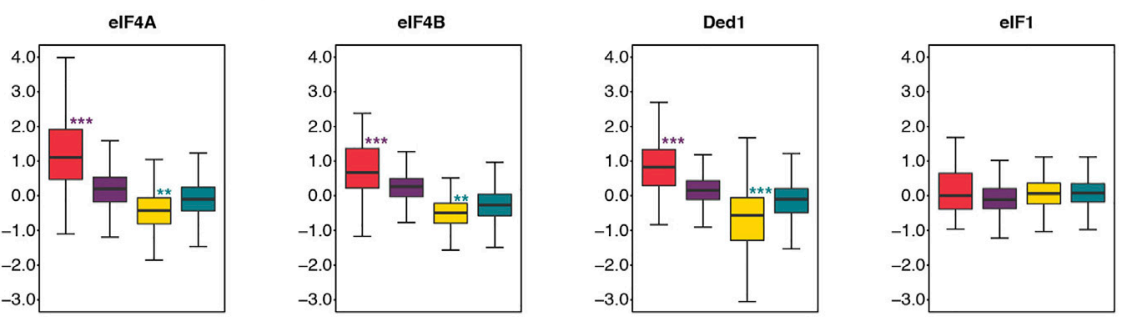

C

$$
\begin{array}{ll}
+\Delta \mathrm{TE}_{\mathrm{rel}} \text { both } & \text { 帛 }-\Delta \mathrm{TE}_{\mathrm{rel}} \text { both } \\
+\Delta \mathrm{TE}_{\mathrm{rel}} \text { Degron only } & \text { 帛 }-\Delta \mathrm{TE}_{\mathrm{rel}} \text { Degron only }
\end{array}
$$

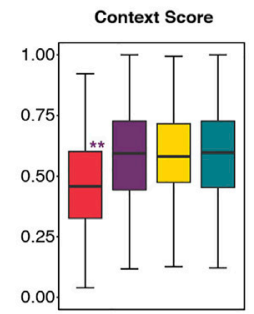

\section{D}

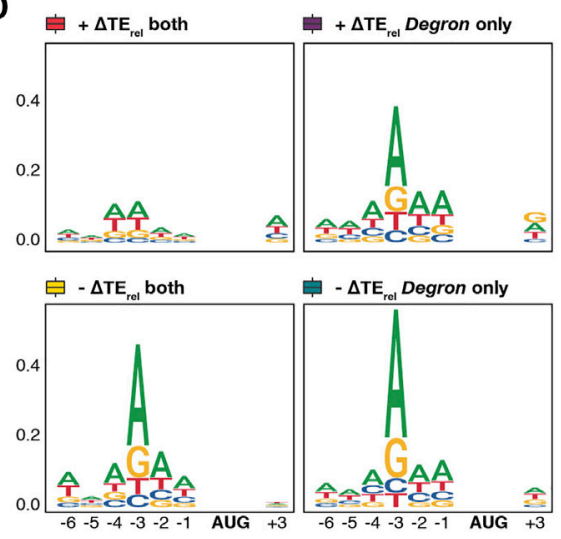

FIGURE 6| The elF3 mRNA-entry-channel arm collaborates with elF4A, elF4B, and Ded1 to drive initiation on mRNAs with long $5^{\prime}$-UTRs and may also discriminate against poor start-codon context. (A) Box and whisker plots comparing different measures of $5^{\prime}$-UTR length or complexity between mRNAs whose TE increases or decreases in both elF3a/b Degron and elF3i DDKK cells (red and yellow, respectively) or increases or decreases only in elF3a/b Degron cells (purple and teal, respectively). ${ }^{\star \star \star}=\mathrm{P}_{\mathrm{adj}}<10^{-10},{ }^{\star \star}=\mathrm{P}_{\mathrm{adj}}<10^{-5},{ }^{*}=\mathrm{P}_{\mathrm{adj}}<0.05$, color indicates comparison set; Wilcoxon Test for $5^{\prime}$-UTR lengths, ANOVA with post-hoc Tukey test for others. (B) Same as in $A$, except comparing $\Delta T_{\text {rel }}$ values observed in cells expressing mutations targeting elF4A, elF4B, Ded1, and elF1. ${ }^{\star \star \star}=P_{\text {adj }}<10^{-10}$, ${ }^{\star \star}=P_{\text {adj }}<$ $10^{-5},{ }^{*}=\mathrm{P}_{\text {adj }}<0.05$, color indicates comparison set; ANOVA with post-hoc Tukey test. (C) Same as in A, except comparing the Kozak sequence context (nt -6 thru +4 ) of affected mRNAs. ${ }^{\star \star \star}=\mathrm{P}_{\text {adj }}<10^{-10},{ }^{\star \star}=\mathrm{P}_{\mathrm{adj}}<10^{-5},{ }^{\star}=\mathrm{P}_{\text {adj }}<0.05$. (D) Sequence logos of $\mathrm{nt}-6$ to +4 of mRNAs whose TE rel either increases (top panels) or decreases (bottom panels) in both elF3a/b Degron and elF3i DDKK cells (left panels) or only in elF3a/b Degron cells (right panels).

closed-loop-dependent mRNAs is particularly sensitive to their disruption.

\section{Comparing the mRNAs Sensitive to Both elF3 Mutations With Those Uniquely Sensitive to the elF3a/b Degron Mutation Provides Clues to the Roles of the elF3i and elF3g Subunits}

Because the eIF3i DDKK and eIF3a/b Degron mutations mimic the loss of either the eIF3i and eIF3g subunits (eIF3i DDKK) or the entire eIF3 complex (eIF3a/b Degron), we reasoned that comparing those mRNAs whose $\mathrm{TE}_{\mathrm{rel}}$ was affected uniquely in $e I F 3 a / b$ Degron cells to those whose $\mathrm{TE}_{\text {rel }}$ was affected in both eIF3a/b Degron and eIF3i DDKK cells might disentangle the roles of these distinct regions of the eIF3 complex. mRNAs whose $\mathrm{TE}_{\mathrm{rel}}$ was affected in both cell lines might depend more heavily on the contributions of the eIF3i and eIF3g subunits of the eIF3 mRNAentry-channel arm, whereas mRNAs whose $\mathrm{TE}_{\text {rel }}$ was affected solely in $e I F 3 a / b$ Degron cells might depend more heavily on the contributions of other eIF3 subunits (or the collaboration of subunits within the intact complex) for their translation. 
To this end, we compared the features of mRNAs whose $\mathrm{TE}_{\mathrm{rel}}$ significantly increased or decreased either in both strains or exclusively in $e I F 3 a / b$ Degron cells (Figure 6A). mRNAs whose $\mathrm{TE}_{\text {rel }}$ decreased in both eIF3i DDKK and eIF3a/b Degron cells possess longer $5^{\prime}$-UTRs than those whose $\mathrm{TE}_{\text {rel }}$ decreased only in eIF3a/b Degron cells, when restricting our analysis to mRNAs previously identified as having a dominant $5^{\prime}$ transcript isoform $\left(\mathrm{P}<10^{-9}\right)$. In contrast, there is no significant difference in the $5^{\prime}$-UTR lengths of mRNAs whose $\mathrm{TE}_{\text {rel }}$ increased. We observed similar results when comparing $5^{\prime}$-UTR lengths reported in a separate study (Kertesz et al., 2010) (Supplementary Figure S5B). We also observed no difference in the degree of structural complexity, as measured by various PARS metrics (Figure 2A), of mRNAs whose $\mathrm{TE}_{\mathrm{rel}}$ was affected either in both eIF3 mutant backgrounds or solely in $e I F 3 a / b$ Degron cells. These results suggest that the eIF3i and eIF3g subunits of the mRNA-entrychannel arm may be specifically required for the contributions of eIF3 to processive scanning through long $5^{\prime}$-UTRs, whereas the other subunits of the eIF3 complex, either independently or in collaboration with eIF3i and eIF3g, participate in its contribution to the resolution of structural impediments during initial mRNA docking and scanning.

We next compared the differential sensitivity of affected mRNAs within these groups to mutations targeting eIF4A, eIF4B, Ded1, or eIF1 (Sen et al., 2015; Sen et al., 2016; Zhou et al., 2020). mRNAs whose $\mathrm{TE}_{\text {rel }}$ increased or decreased in both eIF3i DDKK and eIF3a/b Degron cells were significantly more sensitive to mutations targeting eIF4A, eIF4B, or Ded1 than those uniquely affected in eIF3a/b Degron cells: mRNAs whose $\mathrm{TE}_{\mathrm{rel}}$ decreased in both mutant eIF3 cell lines displayed greater $\mathrm{TE}_{\mathrm{rel}}$ decreases in response to mutations targeting these factors $\left(\mathrm{P}<10^{-9}, \mathrm{P}<10^{-8}\right.$, and $\mathrm{P}<10^{-12}$ for eIF4A, eIF4B, and Ded1, respectively; ANOVA) and mRNAs whose $\mathrm{TE}_{\mathrm{rel}}$ increased displayed stronger $\mathrm{TE}_{\mathrm{rel}}$ increases in these datasets $\left(\mathrm{P}<10^{-16}\right.$ for all comparisons, Figure 6B). In contrast, we observed no significant differences in the relative sensitivity of affected mRNAs to a mutation targeting eIF1. These differential sensitivities are consistent with a role for the mRNA-entry-channel arm in collaborating with eIF4A, eIF4B, and Ded1 during mRNA recruitment.

Finally, mRNAs whose $\mathrm{TE}_{\text {rel }}$ increased in both eIF3i DDKK and $e I F 3 a / b$ Degron cells possess significantly weaker context scores than transcripts whose $\mathrm{TE}_{\text {rel }}$ increased uniquely in eIF3a/b Degron cells $\left(\mathrm{P}<10^{-8}\right.$, Figure 6C). Consistent with this, sequence logos reveal that mRNAs whose $\mathrm{TE}_{\text {rel }}$ increased in both mutant eIF3 cell lines display a weaker preference for adenine at the -3 position (Figure 6D). These observations suggest that eIF3i and eIF3g, and by extension the eIF3 mRNAentry-channel arm, may play a role in discriminating against AUG codons in poor context.

\section{DISCUSSION}

eIF3 is a multisubunit complex that contributes to events throughout the initiation pathway (Hinnebusch, 2006; Valášek et al., 2017). However, disentangling the contributions of eIF3 and its individual subunits to these events has thus far proved challenging.

To shed light on the mechanistic roles of eIF3 and its component subunits, we interrogated the effects of disrupting either the entire eIF3 complex or the eIF3i and eIF3g subunits-both components of the mRNA-entry-channel arm of eIF3 - using ribosome profiling. Our results suggest that the eIF3 complex contributes to driving initiation on mRNAs with long and structurally complex $5^{\prime}$-UTRs and a lower propensity for forming closed-loop structures mediated by eIF4G, eIF4E, and PABP. To a lesser degree, eIF3 may also contribute to discriminating against mRNAs whose start codons appear in weak sequence context. Our results further suggest that eIF3i and eIF3g and thus the eIF3 mRNA-entry-channel arm contribute to the role of eIF3 in facilitating scanning through longer $5^{\prime}$-UTRs, perhaps in collaboration with eIF4A, eIF4B, and Ded1. These subunits may also contribute to discriminating against weak sequence context surrounding the start codon. However, they appear less critical for the role eIF3 plays in resolving structurally complex $5^{\prime}$-UTRs. Instead, the eIF3a, eIF3b, and eIF3c subunits-or all five subunits in collaboration-are required for this role.

Consistent with the strong growth defects provoked by both the eIF3i DDKK and eIF3a/b Degron mutations (Jivotovskaya et al., 2006; Herrmannová et al., 2012), we observed strong global translational defects in the presence of both mutations. Our ribosome profiling results further identified mRNAs in both mutant backgrounds whose $\mathrm{TE}_{\text {rel }}$ was significantly affected, as compared to the overall distribution of $\Delta \mathrm{TE}_{\text {rel }}$ values we observed. Whereas the strong global translational effects we observe suggest that the absolute TE of most mRNAs likely decreases in the presence of both mutations, these absolute effects are removed by the normalization of read counts to library size within each sample. Instead, we focus on the relative changes in $\mathrm{TE}\left(\mathrm{TE}_{\mathrm{rel}}\right)$ we observe in each mRNA, as compared to the overall population of mRNAs across the transcriptome. In the background of global translational suppression that we observe in both cell lines, we interpret these $\mathrm{TE}_{\text {rel }}$ changes as indicating mRNAs whose translation is more dependent (in the case of negative $\Delta \mathrm{TE}_{\mathrm{rel}}$ values) or less dependent (positive $\Delta \mathrm{TE}_{\text {rel }}$ values) than the overall population of mRNAs.

Despite the strong effects on global translation that we observe in both mutant eIF3 strains, we identify many more mRNAs whose $\mathrm{TE}_{\mathrm{rel}}$ is significantly affected in $e I F 3 a / b$ Degron cells. Intriguingly, the set of mRNAs most sensitive $\left(-\Delta \mathrm{TE}_{\text {rel }}\right)$ to the $e I F 3 a / b$ Degron mutation was enriched in mRNAs involved in processes such as mitochondrial translation or metabolism. eIF3 was recently implicated in driving the translation of mitochondrial mRNAs in both fission yeast (Shah et al., 2016) and mammalian cells (Lin et al., 2020), a role which was attributed to the eIF3e and eIF3d subunits. eIF3d has also been linked to the preferential translation of mRNAs involved in cell proliferation pathways in human cells (Lee et al., 2015; Lee et al., 2016). This latter regulatory role appears to involve a capindependent initiation mechanism driven by eIF3. This emerging regulatory role for eIF3 may also be linked to the observation that 
eIF3 binding to the PIC persists through early rounds of elongation in both yeast and mammalian systems (Bohlen et al., 2020; Lin et al., 2020; Wagner et al., 2020). However, neither eIF3d nor eIF3e is present in the budding yeast complex. Our results suggest that the five subunits of the yeast core complex may nonetheless be capable of driving the selective translation of specific mRNAs. More targeted disruption of these subunits might further illuminate the origin of these effects and whether they involve the participation of eIF3 during initiation or early elongation cycles.

In contrast, we did not observe significant enrichment of specific GO terms in the sets of transcripts whose $\mathrm{TE}_{\text {rel }}$ was affected in eIF3i DDKK cells. We further observed a narrower set of transcripts whose translation is affected either more or less strongly than the overall population of mRNAs in the presence of this specific disruption of the eIF3 mRNA-entry-channel arm. That these cells still display strong defects in global translation-and thus likely decreases in the absolute TE of most mRNAs-suggests that the eIF3i and eIF3g subunits might contribute to aspects of mRNA recruitment required more universally across the transcriptome, as opposed to being required to drive translation of specific classes of mRNAs. This was recently suggested for eIF4A in light of the observation that ribosome profiling of cells expressing a temperature-sensitive eIF4A variant that provokes strong global translational defects did not identify substantial numbers of mRNAs more or less sensitive than the overall population, consistent with in vitro measurements suggesting a universal role for eIF4A in alleviating structural complexity within mRNAs (Sen et al., 2015; Yourik et al., 2017).

While our results point to potentially distinct roles for eIF3 and its mRNA-entry-channel arm in either mediating initiation on specific classes of mRNAs or driving the translation of mRNAs across the transcriptome, they also further illuminate the role of eIF3 and its mRNA-entry-channel arm in contributing to mRNA recruitment and its component events of PIC docking, scanning, and start-codon recognition. We show that mRNAs possessing longer $5^{\prime}$-UTRs are more sensitive to disruption of both the entire complex or targeted disruption of the mRNA-entry-channel arm. This is consistent with the identification of mutations throughout the eIF3 complex that affect scanning, with several of these mutations targeting eIF3i (Herrmannová et al., 2012), eIF3g (Cuchalova et al., 2010), and other constituents of the mRNAentry-channel arm (Nielsen et al., 2006; Chiu et al., 2010; Cuchalova et al., 2010).

We also observed that the effects of both eIF3 mutations are similar to those observed via ribosome profiling of cells expressing mutations targeting eIF4A, eIF4B, and Ded1 (Sen et al., 2015; Sen et al., 2016; Zhou et al., 2020), all of which are thought to contribute either to initial PIC docking to the mRNA or subsequent scanning. These similarities extend to the observation that mRNAs likely to form stable closed-loop structures are least sensitive to mutations targeting these initiation factors and to both eIF3 mutations, whereas mRNAs less likely to form stable closed-loop structures are more sensitive. Together, these observations suggest that eIF3 may collaborate with eIF4A, eIF4B, and Ded1 to drive initiation on mRNAs with longer $5^{\prime}$-UTRs and a weaker dependence on the initiation factors eIF4G, eIF4E, and PABP. eIF3 - via the eIF3a CTD component of the eIF3 mEnC arm-interacts with eIF4B (Methot et al., 1996) and with the 40S latch (Chiu et al., 2010). Moreover, eIF3 is present in both the mRNA-entry and -exit channels of the ribosome in both yeast and mammalian structures (Aylett et al., 2015; Simonetti et al., 2016). In a recent structure of the human $48 \mathrm{~S}$ complex, eIF3g was observed binding to ribosomal RNA and protein elements within the mRNA-entry channel and eIF3k, eIF3l, and eIF3e were found adjacent to eIF4A near the mRNA-exit channel (Querido et al., 2020). And yet, eIF3k, -l, and -e are absent from the yeast eIF3 complex, where eIF3a and eIF3c are alone found near the mRNA-exit channel of the ribosome. However, in vitro studies reveal that, in addition to eIF3d and eIF3e, eIF3c (which is present in budding yeast) is also able to bind to components of the eIF4F complex (Villa et al., 2013). Together with our results here, these observations together raise several possibilities for direct or functional collaboration between eIF3 and these other factors.

eIF3 has also been implicated in driving cap-independent initiation mechanisms (Lee et al., 2016; Rode et al., 2018; Bhardwaj et al., 2019) and in directly recruiting the PIC to specific classes of mRNAs (Lee et al., 2015; Lee et al., 2016; Shah et al., 2016; Lin et al., 2020; Lamper et al., 2020), both of which may circumvent the requirement for eIF4G-, eIF4E-, and PABP-mediated closed-loop formation. Our observation that these effects manifest upon targeting either the entire eIF3 complex or simply the mRNA-entry-channel arm point to a role for the mRNA-entry-channel arm in collaborating with these other factors to mediate initiation in the absence of stable closedloop formation. In fact, eIF3 and eIF4A were recently shown to collaborate in a non-canonical initiation pathway that circumvents eIF4E and eIF4G during neuronal development in Drosophila (Rode et al., 2018).

In contrast, we observed stronger effects of mRNA structural complexity when disrupting the entire eIF3 complex than when specifically targeting its mRNA-entry-channel arm. Certainly, this does not exclude the possibility that either or both eIF3i and eIF3g contribute to resolving regions of structural complexity within the $5^{\prime}$-UTR. Mutations targeting these subunits have previously been shown to interfere with initiation on reporter mRNAs containing stable stem loop structures (Cuchalova et al., 2010; Herrmannová et al., 2012). Nonetheless, our results point to the remaining eIF3 subunits-eIF3a, eIF3b, and eIF3c, either alone or in collaboration with eIF3i and eIF3g-playing a role in resolving structural complexity during initial PIC docking or scanning. Both eIF3a (via its CTD) and eIF3b contribute to the mRNA-entry-channel arm (Aylett et al., 2015; Llácer et al., 2015; Simonetti et al., 2016; Llácer et al., 2018) and mutations targeting the eIF3a CTD disrupt initiation on reporter mRNAs containing stem loop structures (Chiu et al., 2010). In addition, eIF3a interacts physically and functionally with mRNA near the mRNA-exit channel of the ribosome (Szamecz et al., 2008; Munzarová et al., 2011; Aitken et al., 2016; Llácer et al., 2018) and eIF3c binds components of the eIF4F complex (Villa et al., 2013) that have recently been visualized near the mRNA-exit channel in the human 48S PIC (Querido et al., 2020). 
Whereas we observed relatively strong effects on either initial docking or scanning in the presence of both eIF3 mutations, we observed more nuanced effects on start-codon recognition upon disruption of the eIF3 mRNA-entry-channel arm. In contrast to the similarities between the effects of both eIF3 mutations and those previously observed for mutations targeting eIF4A, eIF4B, and Ded1, we observed relatively little similarity with those observed in previous ribosome profiling experiments targeting eIF1. Nonetheless, our observation that those mRNAs least sensitive to the eIF3i DDKK mutation exhibit weaker startcodon context does suggest that the eIF3 mRNA-entrychannel arm plays a role in discriminating against AUG codons in weak context. Consistent with this, mutations targeting several components of the mRNA-entry-channel arm elicit defects in either the accuracy or efficiency of start-codon recognition (Nielsen et al., 2004, 2006; Chiu et al., 2010; Cuchalova et al., 2010; Herrmannová et al., 2012). The fact that we observe these modest effects but do not observe similarities between our dataset and ribosome profiling data from eIF1 mutant cells suggests that these effects do not arise from disruption of the functional collaboration between eIF3 and eIF1(Valasek et al., 2004; Llácer et al., 2015; Llácer et al., 2018). Instead, it is possible that disruption of the eIF3 mRNA-entrychannel arm might disrupt its modulation of the equilibrium between the open and closed states of the PIC via interaction with the $40 \mathrm{~S}$ latch. Indeed, mutations targeting this nexus produce start-codon recognition defects (Chiu et al., 2010; Dong et al., 2017).

Overall, we observed striking similarities between the effects of the eIF3i DDKK and eIF3a/b Degron mutations. Consistent with their disruption of either a portion of the eIF3 mRNA-entrychannel arm $(e I F 3 i D D K K)$ or the entire complex $(e I F 3 a / b$ Degron), the affected mRNAs we identified in eIF3i DDKK cells comprise subsets of those we identified in $e I F 3 a / b$ Degron cells. Nonetheless, comparison of those transcripts whose $\mathrm{TE}_{\mathrm{rel}}$ was affected in both eIF3 mutant backgrounds with those affected solely in $e I F 3 a / b$ Degron cells identifies telling differences that suggest roles for the eIF3i and eIF3g subunits of the eIF3 mRNAentry-channel arm. mRNAs whose $\mathrm{TE}_{\mathrm{rel}}$ decreases in both backgrounds-suggesting that their translation depends more strongly on the eIF3i and eIF3g subunits affected by both mutations-have $5^{\prime}$-UTRs that are longer but are no more structurally complex than those whose $\mathrm{TE}_{\text {rel }}$ decreases only when the entire eIF3 complex is disrupted. mRNAs sensitive to both mutations are also more strongly affected by mutations targeting eIF4A, eIF4B, and Ded1. mRNAs whose $\mathrm{TE}_{\text {rel }}$ decreased in both backgrounds displayed stronger $\mathrm{TE}_{\text {rel }}$ decreases in the presence of these other mutations than mRNAs whose $\mathrm{TE}_{\mathrm{rel}}$ decreased solely in eIF3a/b Degron cells. mRNAs whose $\mathrm{TE}_{\mathrm{rel}}$ increased in both mutant eIF3 backgrounds similarly display stronger $\mathrm{TE}_{\text {rel }}$ increases in cells expressing mutants of eIF4A, eIF4B, or Ded1. mRNAs whose $\mathrm{TE}_{\text {rel }}$ increased in both eIF3 backgrounds further possess weaker start-codon sequence context than those whose $\mathrm{TE}_{\text {rel }}$ increased solely in $e I F 3 a / b$ Degron cells.

Together, these observations again point to roles for eIF3i and eIF3g, and by extension the eIF3 mRNA-entry-channel arm, in functional collaboration with eIF4A, eIF4B, and Ded1 to drive processive scanning through longer $5^{\prime}$-UTRs on mRNAs whose translation is less dependent on the formation of a stable closed loop. Surprisingly, effects on scanning processivity were not previously observed using a set of reporter constructs in extracts derived from cells expressing eIF3i DDKK cells (Herrmannová et al., 2012), perhaps because other features present in natural mRNAs or mRNPs are required to elicit these effects. Distinct mutations targeting either eIF3i or eIF3g, however, do manifest defects in scanning processivity (Cuchalova et al., 2010). Our results also suggest that eIF3i and eIF3g play a role in discriminating against weak start-codon context during start-codon recognition. Previous experiments following the effects of the eIF3i DDKK mutation using a series of reporter mRNAs observed leaky scanning of AUG codons, suggesting these subunits might contribute to efficient start-codon recognition (Herrmannová et al., 2012). However, the effect of codon context was not reported.

While the eIF3i and eIF3g subunits of the eIF3 mRNA-entrychannel arm appear to contribute to scanning processivity, the remaining subunits (or the entire complex) appear to contribute to resolving structural complexity within $5^{\prime}$-UTRs. We observed stronger and more significant effects of various measures of structural complexity throughout the $5^{\prime}$-UTRs of mRNAs in $e I F 3 a / b$ Degron cells than we did in eIF3i DDKK cells. That we do not observe these effects in eIF3i DDKK cells but still observe strong similarities with those effects observed in mutant eIF4A, eIF4B, or Ded 1 cells (and find that mRNAs sensitive only to the $e I F 3 a / b$ Degron mutation are less sensitive to mutations targeting these factors) might suggest that eIF3 can also contribute to the resolution of structural complexity independently of any collaboration with these factors. Another possibility is that eIF3 does indeed collaborate with these factors but via distinct functional mechanisms to resolve stable structural impediments near the $5^{\prime}$ cap or within downstream $5^{\prime}$-UTR regions.

Finally, we also found that mRNAs whose translation is most sensitive to the disruption of the entire eIF3 complex were enriched in mRNAs involved in mitochondrial translation and metabolism. This observation echoes the recently identified role of eIF3 in preferentially mediating translation on these classes of mRNAs in fission yeast and mammalian cells (Shah et al., 2016; Lin et al., 2020). That role appears to involve the eIF3d subunit, which has also been implicated in mediating the translation of mRNAs involved in cell proliferation (Lee et al., 2016), as well as the ability of eIF3 to remain bound during early rounds of elongation (Bohlen et al., 2020; Lin et al., 2020; Wagner et al., 2020). Because eIF3d is absent in budding yeast cells, our observations suggest that at least one subunit of the core complex is capable of reprising aspects of this role. A potential candidate is eIF3a which, like eIF3d, is positioned near the mRNA-exit channel of the ribosome and appears to interact physically and functionally with the mRNA there (Szamecz et al., 2008; Aitken et al., 2016; Llácer et al., 2018). eIF3a has also previously been implicated in mediating sequence-dependent reinitiation events, a function 
which requires direct interaction with specific mRNA sequence elements (Szamecz et al., 2008; Munzarová et al., 2011). The mechanistic origin of these effects in budding yeast, and whether they involve the participation of eIF3 during initiation, early rounds of elongation, or both, emerge as intriguing questions.

Our work sheds light on the specific roles of the eIF3 mRNAentry-channel arm and its other subunits during the component events of mRNA recruitment. It further points to a potential role for eIF3 in mediating the translation of specific classes of mRNAs, as in higher eukaryotic cells. Nonetheless, experiments following the fate of reporter constructs containing the $5^{\prime}$-UTRs of sensitive mRNAs in eIF3i DDKK and eIF3a/b Degron cells or cell extracts or the requirements of sensitive mRNAs for eIF3 or eIF3i and eIF3g in mRNA recruitment assays in vitro might further strengthen the case for these roles. Still further investigation is necessary to determine the mechanism whereby eIF3 mediates translation of these mRNAs, how it functions to facilitate initiation on mRNAs with structurally complex $5^{\prime}$-UTRs, and how its mRNA-entry-channel arm collaborates with other initiation factors to drive initial docking and scanning on mRNAs independent of closed-loop formation.

\section{DATA AVAILABILITY STATEMENT}

Sequencing data from this study have been submitted to the NCBI Gene Expression Omnibus (GEO; http://www.ncbi.nlm.nih.gov/ geo/) under accession number GSE190601.

\section{AUTHOR CONTRIBUTIONS}

CA performed the experiments, wrote the manuscript, prepared final versions of the figures, and performed data analysis. AS performed significant data preparation, analysis, and interpretation. JL performed data analysis and interpretation. LF and SGH performed data analysis. SK performed sample preparation and data preparation.

\section{FUNDING}

CEA is supported by R15 GM140372 (NIH/NIGMS). AS is an undergraduate student in the Computer Science Department at Vassar College. JL is an undergraduate student in the Biochemistry Program at Vassar College; LF and SGH are undergraduate students in the Biology Department at Vassar College and are supported by R15 GM140372 (NIH/NIGMS).

\section{ACKNOWLEDGMENTS}

CEA would like to acknowledge Jon R. Lorsch and Alan G. Hinnebusch of the National Institutes of Health for their support and guidance during the initial phases of this project. In addition,
CEA would like to acknowledge Jennifer Kennell and Zachary Donhauser of the Vassar College Biochemistry Program, Justin Touchon of the Vassar College Biology Department, and Shardul Kulkarni of the Department of Biochemistry \& Molecular Biology at Penn State Eberly College of Science for their comments and guidance during the preparation of this manuscript.

\section{SUPPLEMENTARY MATERIAL}

The Supplementary Material for this article can be found online at: https://www.frontiersin.org/articles/10.3389/fmolb.2021.787664/ full\#supplementary-material

Supplementary Figure 1 | Disruption of the elF3 complex or its mRNA-entrychannel arm provoke strong global translational defects. (A) Polysome profiles collected from elF3a/b Degron and elF3i DDKK cells and isogenic wild-type cells grown under permissive conditions. (B,C) Polysome profiles collected from biological replicates used to prepare ribosome profiling libraries for elF3i DDKK (B) and elF3a/b Degron (C) cells and isogenic wild-type cells upon shifting to growth under restrictive conditions for $30 \mathrm{~min}$ (elF3i DDKK) or $90 \mathrm{~min}$ (elF3a/b Degron).

Supplementary Figure 2 | mRNA fragment and Ribosome footprint libraries obtained from biological replicates of elF3i DDKK and elF3a/b Degron cells are highly reproducible. Correlation plots of read counts obtained from mRNA fragment and ribosome footprint libraries prepared from biological replicates of elF3i DDKK and elF3a/b Degron cells and matching isogenic wild-type cells.

Supplementary Figure 3 | Disruption of the elF3 complex affects the translation of mRNAs associated with mitochondrial processes. Bar plots showing the percent over- or under-representation of specific biological process GO terms in the list of mRNAs whose $\mathrm{TE}_{\text {rel }}$ either significantly decreases (left) or increases (right) in elF3a/b Degron cells. Color scale indicates $\mathrm{P}_{\text {adj }}$ values.

Supplementary Figure $\mathbf{4}$ | Disruption of elF3 or its mRNA-entry-channel arm result in global increases in relative UORF translation that are strongest for UORFs closest to the 5' end. (A) Correlation of UORF TE $E_{\text {rel }}$ values observed in either elF3a/b Degron (left) or elF3i DDKK (right) cells and those observed in isogenic wild-type cells. UORFs displaying significant $\left(\mathrm{P}_{\mathrm{adj}}<0.05\right) \mathrm{TE}_{\mathrm{rel}}$ increases $(>50 \%$ red, $>100 \%$ blue $)$ or decreases ( $>50 \%$ yellow, $>100 \%$ green) as determined by DESeq2 analysis are shown in colors. (B) Correlation of observed $\triangle \mathrm{TE}_{\mathrm{rel}}$ values and distance from the ${ }^{\prime}$ end for individual uORFs in elF3a/b Degron (left) and elF3i DDKK (right) cells. Spearman correlation fits shown in red.

Supplementary Figure $\mathbf{5}$ | Disruption of elF3 or its mRNA-entry-channel arm affect the translation of mRNAs with long 5'-UTRs. (A) Box and whisker plots comparing 5'-UTR lengths (reported by Kertesz, et al., Nature 2010) between mRNAs whose $T_{E_{\text {rel }}}$ significantly increases (red), decreases (yellow), or is unaffected (white) in each elF3 mutant strain. ${ }^{\star \star \star}=P_{\text {adj }}<10^{-10},{ }^{\star \star}=P_{\text {adj }}<10$ $5,{ }^{*}=P_{\text {adj }}<0.05$, color indicates comparison set; Wilcoxon test. (B) Same as in A, except comparing 5'-UTR lengths between mRNAs whose $\mathrm{TE}_{\text {rel }}$ significantly increases or decreases in both elF3a/b Degron and elF3i DDKK cells (red and yellow, respectively) or increases or decreases only in elF3a/b Degron cells (purple and teal, respectively). ${ }^{\star \star \star}=\mathrm{P}_{\mathrm{adj}}<10^{-10},{ }^{\star \star}{ }^{\star}=\mathrm{P}_{\mathrm{adj}}<10^{-5},{ }^{\star}=\mathrm{P}_{\mathrm{adj}}<0.05$, color indicates comparison set; Wilcoxon test.

Supplementary Figure 6 | Observed $\Delta \mathrm{TE}_{\mathrm{rel}}$ values in elF3a/b Degron and elF3i $D D K K$ cells are not correlated with the AUG sequence context of individual mRNAs. Correlation plots comparing observed $\triangle \mathrm{TE}_{\text {rel }}$ values in elF3a/b Degron (left) or elF3i $D D K K$ (right) cells and the AUG sequence context (nt -6 to +4 ) of individual mRNAs. Predicted Spearman correlations shown in red.

Supplementary Figure $7 \mid \Delta \mathrm{TE}_{\text {rel }}$ values observed in elF3a/b Degron and elF3 $D D K K$ cells strongly correlate with those observed in the presence of mutations targeting elF4A, elF4B, and Ded1 but not elF1 or elF1A. Correlation plots comparing observed $\triangle T E_{\text {rel }}$ values in elF3a/b Degron (top) or elF3i DDKK (bottom) cells and those observed for mutations targeting elF4A, elF4B, Ded1, elF1, and elF1A. Predicted Spearman correlations shown in red. 


\section{REFERENCES}

Aitken, C. E., Beznosková, P., Vlčkova, V., Chiu, W.-L., Zhou, F., Valášek, L. S., et al. (2016). Eukaryotic Translation Initiation Factor 3 Plays Distinct Roles at the Mrna Entry and Exit Channels of the Ribosomal Preinitiation Complex. Elife 5, 1. doi:10.7554/eLife.20934

Aitken, C. E., and Lorsch, J. R. (2012). A Mechanistic Overview of Translation Initiation in Eukaryotes. Nat. Struct. Mol. Biol. 19, 568-576. doi:10.1038/nsmb.2303

Asano, K., Clayton, J., Shalev, A., and Hinnebusch, A. G. (2000). A Multifactor Complex of Eukaryotic Initiation Factors, eIF1, eIF2, eIF3, eIF5, and Initiator tRNAMet Is an Important Translation Initiation Intermediate In Vivo. Genes Dev. 14, 2534-2546. doi:10.1101/gad.831800

Aylett, C. H. S., Boehringer, D., Erzberger, J. P., Schaefer, T., and Ban, N. (2015). Structure of a Yeast 40S-eIF1-eIF1A-eIF3-eIF3j Initiation Complex. Nat. Struct. Mol. Biol. 22, 269-271. doi:10.1038/nsmb.2963

Bhardwaj, U., Powell, P., and Goss, D. J. (2019). Eukaryotic Initiation Factor (eIF) 3 Mediates Barley Yellow Dwarf Viral mRNA $3^{\prime}-5^{\prime}$ UTR Interactions and $40 \mathrm{~S}$ Ribosomal Subunit Binding to Facilitate Cap-independent Translation. Nucleic Acids Res. 47, 6225-6235. doi:10.1093/nar/gkz448

Bohlen, J., Fenzl, K., Kramer, G., Bukau, B., and Teleman, A. A. (2020). Selective 40S Footprinting Reveals Cap-Tethered Ribosome Scanning in Human Cells. Mol. Cel. 79, 561-574. doi:10.1016/j.molcel.2020.06.005

Brito Querido, J., Sokabe, M., Kraatz, S., Gordiyenko, Y., Skehel, J. M., Fraser, C. S., et al. (2020). Structure of a Human $48 \mathrm{~S}$ Translational Initiation Complex. Science 369, 1220-1227. doi:10.1126/SCIENCE.ABA4904

Chiu, W.-L., Wagner, S., Herrmannova', A., Burela, L., Zhang, F., Saini, A. K., et al. (2010). The C-Terminal Region of Eukaryotic Translation Initiation Factor 3a (eIF3a) Promotes mRNA Recruitment, Scanning, and, Together with eIF3j and the eIF3b RNA Recognition Motif, Selection of AUG Start Codons. Mol. Cell. Biol. 30, 4415-4434. doi:10.1128/mcb.00280-10

Costello, J., Castelli, L. M., Rowe, W., Kershaw, C. J., Talavera, D., MohammadQureshi, S. S., et al. (2015). Global mRNA Selection Mechanisms for Translation Initiation. Genome Biol. 16, 1. doi:10.1186/s13059-014-0559-z

Cuchalova', L., Kouba, T., Herrmannova', A., Da'nyi, I., Chiu, W.-l., Vala'šek, L., et al. (2010). The RNA Recognition Motif of Eukaryotic Translation Initiation Factor 3g (eIF3g) Is Required for Resumption of Scanning of Posttermination Ribosomes for Reinitiation on GCN4 and Together with eIF3i Stimulates Linear Scanning. Mol. Cell. Biol. 30, 4671-4686. doi:10.1128/mcb.00430-10

Des Georges, A., Dhote, V., Kuhn, L., Hellen, C. U. T., Pestova, T. V., Frank, J., et al. (2015). Structure of Mammalian eIF3 in the Context of the 43S Preinitiation Complex. Nature 525, 491-495. doi:10.1038/nature14891

Dong, J., Aitken, C. E., Thakur, A., Shin, B.-S., Lorsch, J. R., and Hinnebusch, A. G. (2017). Rps3/uS3 Promotes mRNA Binding at the 40S Ribosome Entry Channel and Stabilizes Preinitiation Complexes at Start Codons. Proc. Natl. Acad. Sci. USA 114, E2126-E2135. doi:10.1073/pnas.1620569114

Elantak, L., Wagner, S., Herrmannová, A., Karásková, M., Rutkai, E., Lukavsky, P. J., et al. (2010). The Indispensable N-Terminal Half of eIF3j/HCR1 Cooperates with its Structurally Conserved Binding Partner eIF3b/PRT1-RRM and with eIF1A in Stringent AUG Selection. J. Mol. Biol. 396, 1097-1116. doi:10.1016/j.jmb.2009.12.047

Gerashchenko, M. V., and Gladyshev, V. N. (2014). Translation Inhibitors Cause Abnormalities in Ribosome Profiling Experiments. Nucleic Acids Res. 42, e134. doi:10.1093/NAR/GKU671

Gupta, N., Lorsch, J. R., and Hinnebusch, A. G. (2018). Yeast Ded1 Promotes $48 S$ Translation Pre-initiation Complex Assembly in an mRNA-specific and eIF4Fdependent Manner. Elife 7, 1. doi:10.7554/eLife.38892

Herrmannová, A., Daujotytė, D., Yang, J.-C., Cuchalová, L., Gorrec, F., Wagner, S., et al. (2012). Structural Analysis of an eIF3 Subcomplex Reveals Conserved Interactions Required for a Stable and Proper Translation Pre-initiation Complex Assembly. Nucleic Acids Res. 40, 2294-2311. doi:10.1093/nar/gkr765

Hinnebusch, A. G. (2006). eIF3: a Versatile Scaffold for Translation Initiation Complexes. Trends Biochem. Sci. 31, 553-562. doi:10.1016/j.tibs.2006.08.005

Hinnebusch, A. G., Ivanov, I. P., and Sonenberg, N. (2016). Translational Control by $5^{\prime}$-untranslated Regions of Eukaryotic mRNAs. Science 352, 1413-1416. doi: $10.1126 /$ science.aad 9868

Hinnebusch, A. G. (2017). Structural Insights into the Mechanism of Scanning and Start Codon Recognition in Eukaryotic Translation Initiation. Trends Biochem. Sci. 42, 589-611. doi:10.1016/j.tibs.2017.03.004
Hinnebusch, A. G. (2014). The Scanning Mechanism of Eukaryotic Translation Initiation. Annu. Rev. Biochem. 83, 779-812. doi:10.1146/annurev-biochem060713-035802

Ingolia, N. T. (2010). Genome-Wide Translational Profiling by Ribosome Footprinting. Methods Enzymol. 470, 119-142. doi:10.1016/S0076-6879(10) 70006-9

Ingolia, N. T., Ghaemmaghami, S., Newman, J. R. S., and Weissman, J. S. (2009). Genome-wide Analysis In Vivo of Translation with Nucleotide Resolution Using Ribosome Profiling. Science 324, 218-223. doi:10.1126/science.1168978

Jackson, R. J., Hellen, C. U. T., and Pestova, T. V. (2010). The Mechanism of Eukaryotic Translation Initiation and Principles of its Regulation. Nat. Rev. Mol. Cel. Biol. 11, 113-127. doi:10.1038/nrm2838

Jivotovskaya, A. V., Vala'šek, L., Hinnebusch, A. G., and Nielsen, K. H. (2006). Eukaryotic Translation Initiation Factor 3 (eIF3) and eIF2 Can Promote mRNA Binding to 40 S Subunits Independently of eIF4G in Yeast. Mol. Cell. Biol. 26, 1355-1372. doi:10.1128/mcb.26.4.1355-1372.2006

Kertesz, M., Wan, Y., Mazor, E., Rinn, J. L., Nutter, R. C., Chang, H. Y., et al. (2010). Genome-wide Measurement of RNA Secondary Structure in Yeast. Nature 467, 103-107. doi:10.1038/nature09322

Kulkarni, S. D., Zhou, F., Sen, N. D., Zhang, H., Hinnebusch, A. G., and Lorsch, J. R. (2019). Temperature-dependent Regulation of Upstream Open reading Frame Translation in S. cerevisiae. BMC Biol. 17, 1. doi:10.1186/s12915-019-0718-5

Lamper, A. M., Fleming, R. H., Ladd, K. M., and Lee, A. S. Y. (2020). A Phosphorylation-Regulated eIF3d Translation Switch Mediates Cellular Adaptation to Metabolic Stress. Science 370 (6518), 1853-856. doi:10.1126/ science.abb0993

Lee, A. S. Y., Kranzusch, P. J., and Cate, J. H. D. (2015). EIF3 Targets CellProliferation Messenger RNAs for Translational Activation or Repression. Nature 522, 111-114. doi:10.1038/nature14267

Lee, A. S. Y., Kranzusch, P. J., Doudna, J. A., and Cate, J. H. D. (2016). EIF3d Is an mRNA Cap-Binding Protein that Is Required for Specialized Translation Initiation. Nature 536, 96-99. doi:10.1038/nature18954

Lin, Y., Li, F., Huang, L., Polte, C., Duan, H., Fang, J., et al. (2020). eIF3 Associates with $80 \mathrm{~S}$ Ribosomes to Promote Translation Elongation, Mitochondrial Homeostasis, and Muscle Health. Mol. Cel. 79, 575-587. doi:10.1016/ j.molcel.2020.06.003

Llácer, J. L., Hussain, T., Dong, J., Villamayor, L., Gordiyenko, Y., and Hinnebusch, A. G. (2021). Large-Scale Movement of eIF3 Domains During Translation Initiation Modulate Start Codon Selection. Nucleic Acids Research 49 (20), 11491-11511. doi:10.1093/nar/gkab908

Llácer, J. L., Hussain, T., Marler, L., Aitken, C. E., Thakur, A., Lorsch, J. R., et al. (2015). Conformational Differences between Open and Closed States of the Eukaryotic Translation Initiation Complex. Mol. Cel. 59, 399-412. doi:10.1016/ j.molcel.2015.06.033

Llácer, J. L., Hussain, T., Saini, A. K., Nanda, J. S., Kaur, S., Gordiyenko, Y., et al. (2018). Translational Initiation Factor eIF5 Replaces IF1 on the 40S Ribosomal Subunit to Promote Start-Codon Recognition. Elife 7, 1. doi:10.7554/ ELIFE.39273

Love, M. I., Huber, W., and Anders, S. (2014). Moderated Estimation of Fold Change and Dispersion for RNA-Seq Data with DESeq2. Genome Biol. 15, 550. doi:10.1186/s13059-014-0550-8

Majumdar, R., Bandyopadhyay, A., and Maitra, U. (2003). Mammalian Translation Initiation Factor eIF1 Functions with eIF1A and eIF3 in the Formation of a Stable 40 S Preinitiation Complex. J. Biol. Chem. 278, 6580-6587. doi:10.1074/ jbc.m210357200

Martin-Marcos, P., Zhou, F., Karunasiri, C., Zhang, F., Dong, J., Nanda, J., et al. (2017). eIF1A Residues Implicated in Cancer Stabilize Translation Preinitiation Complexes and Favor Suboptimal Initiation Sites in Yeast. Elife 6, 1 . doi:10.7554/eLife.31250

Méthot, N., Song, M. S., and Sonenberg, N. (1996). A Region Rich in Aspartic Acid, Arginine, Tyrosine, and glycine (DRYG) Mediates Eukaryotic Initiation Factor 4B (eIF4B) Self-Association and Interaction with eIF3. Mol. Cell. Biol. 16, 5328-5334. doi:10.1128/mcb.16.10.5328

Mitchell, S. F., Walker, S. E., Algire, M. A., Park, E.-H., Hinnebusch, A. G., and Lorsch, J. R. (2010). The 5'-7-Methylguanosine Cap on Eukaryotic mRNAs Serves Both to Stimulate Canonical Translation Initiation and to Block an Alternative Pathway. Mol. Cel. 39, 950-962. doi:10.1016/ j.molcel.2010.08.021 
Munzarová, V., Pánek, J., Gunišová, S., Dányi, I., Szamecz, B., and Valášek, L. S. (2011). Translation Reinitiation Relies on the Interaction between eIF3a/TIF32 and Progressively Folded Cis-Acting mRNA Elements Preceding Short uORFs. Plos Genet. 7, e1002137. doi:10.1371/journal.pgen.1002137

Nielsen, K. H., Szamecz, B., Valášek, L., Jivotovskaya, A., Shin, B.-S., and Hinnebusch, A. G. (2004). Functions of eIF3 Downstream of 48S Assembly Impact AUG Recognition and GCN4 Translational Control. EMBO J. 23, 1166-1177. doi:10.1038/sj.emboj.7600116

Nielsen, K. H., Vala'šek, L., Sykes, C., Jivotovskaya, A., and Hinnebusch, A. G. (2006). Interaction of the RNP1 Motif in PRT1 with HCR1 Promotes 40S Binding of Eukaryotic Initiation Factor 3 in Yeast. Mol. Cell. Biol. 26, 2984-2998. doi:10.1128/mcb.26.8.2984-2998.2006

Pelechano, V., Wei, W., and Steinmetz, L. M. (2013). Extensive Transcriptional Heterogeneity Revealed by Isoform Profiling. Nature 497, 127-131. doi:10.1038/nature12121

Rode, S., Ohm, H., Anhäuser, L., Wagner, M., Rosing, M., Deng, X., et al. (2018). Differential Requirement for Translation Initiation Factor Pathways during Ecdysone-dependent Neuronal Remodeling in Drosophila. Cel. Rep. 24, 2287-2299. e4. doi:10.1016/J.CELREP.2018.07.074

Sen, N. D., Gupta, N., K. Archer, S., Preiss, T., Lorsch, J. R., and Hinnebusch, A. G. (2019). Functional Interplay between DEAD-Box RNA Helicases Ded1 and Dbp1 in Preinitiation Complex Attachment and Scanning on Structured mRNAs In Vivo. Nucleic Acids Res. 47, 8785-8806. doi:10.1093/nar/gkz595

Sen, N. D., Zhou, F., Harris, M. S., Ingolia, N. T., and Hinnebusch, A. G. (2016). eIF4B Stimulates Translation of Long mRNAs with Structured $5^{\prime}$ UTRs and Low Closed-Loop Potential but Weak Dependence on eIF4G. Proc. Natl. Acad. Sci. USA 113, 10464-10472. doi:10.1073/pnas.1612398113

Sen, N. D., Zhou, F., Ingolia, N. T., and Hinnebusch, A. G. (2015). Genome-wide Analysis of Translational Efficiency Reveals Distinct but Overlapping Functions of Yeast DEAD-Box RNA Helicases Ded1 and eIF4A. Genome Res. 25, 1196-1205. doi:10.1101/gr.191601.115

Sha, Z., Brill, L. M., Cabrera, R., Kleifeld, O., Scheliga, J. S., Glickman, M. H., et al. (2009). The eIF3 Interactome Reveals the Translasome, a Supercomplex Linking Protein Synthesis and Degradation Machineries. Mol. Cel. 36, 141-152. doi:10.1016/j.molcel.2009.09.026

Shah, M., Su, D., Scheliga, J. S., Pluskal, T., Boronat, S., Motamedchaboki, K., et al. (2016). A Transcript-specific eIF3 Complex Mediates Global Translational Control of Energy Metabolism. Cel. Rep. 16, 1891-1902. doi:10.1016/ J.CELREP.2016.07.006

Shivaya Valasek, L. (2012). 'Ribozoomin' - Translation Initiation from the Perspective of the Ribosome-Bound Eukaryotic Initiation Factors (eIFs). Cpps 13, 305-330. doi:10.2174/138920312801619385

Simonetti, A., Brito Querido, J., Myasnikov, A. G., Mancera-Martinez, E., Renaud, A., Kuhn, L., et al. (2016). eIF3 Peripheral Subunits Rearrangement after mRNA Binding and Start-Codon Recognition. Mol. Cel. 63, 206-217. doi:10.1016/ j.molcel.2016.05.033

Sokabe, M., and Fraser, C. S. (2014). Human Eukaryotic Initiation Factor 2 (eIF2)GTP-Met-tRNAi Ternary Complex and eIF3 Stabilize the 43 S Preinitiation Complex. J. Biol. Chem. 289, 31827-31836. doi:10.1074/JBC.M114.602870

Sonenberg, N., and Hinnebusch, A. G. (2009). Regulation of Translation Initiation in Eukaryotes: Mechanisms and Biological Targets. Cell 136, 731-745. doi:10.1016/j.cell.2009.01.042

Szamecz, B., Rutkai, E., Cuchalová, L., Munzarová, V., Herrmannová, A., Nielsen, K. H., et al. (2008). eIF3a Cooperates with Sequences $5^{\prime}$ of uORF1 to Promote Resumption of Scanning by post-termination Ribosomes for Reinitiation on GCN4 mRNA. Genes Dev. 22, 2414-2425. doi:10.1101/ gad. 480508
Valásek, L., Mathew, A. A., Shin, B. S., Nielsen, K. H., Szamecz, B., and Hinnebusch, A. G. (2003). The Yeast eIF3 Subunits TIF32/a, NIP1/c, and eIF5 Make Critical Connections with the 40S Ribosome In Vivo. Genes Dev. 17, 786-799. doi:10.1101/gad.1065403

Valásek, L., Nielsen, K. H., Zhang, F., Fekete, C. A., and Hinnebusch, A. G. (2004). Interactions of Eukaryotic Translation Initiation Factor 3 (eIF3) Subunit NIP1/ c with eIF1 and eIF5 Promote Preinitiation Complex Assembly and Regulate Start Codon Selection. Mol. Cell. Biol. 24, 9437-9455. doi:10.1128/ MCB.24.21.9437-9455.2004

Valášek, L., Nielsen, K. H., and Hinnebusch, A. G. (2002). Direct eIF2-eIF3 Contact in the Multifactor Complex Is Important for Translation Initiation In Vivo. EMBO J. 21, 5886-5898. doi:10.1093/emboj/cdf563

Valášek, L. S., Zeman, J., Wagner, S., Beznosková, P., Pavlíková, Z., Mohammad, M. P., et al. (2017). Embraced by eIF3: Structural and Functional Insights into the Roles of eIF3 across the Translation Cycle. Nucleic Acids Res. 45, 10948-10968. doi:10.1093/nar/gkx805

Villa, N., Do, A., Hershey, J. W. B., and Fraser, C. S. (2013). Human Eukaryotic Initiation Factor 4G (eIF4G) Protein Binds to eIF3c, -d, and -e to Promote mRNA Recruitment to the Ribosome. J. Biol. Chem. 288, 32932-32940. doi:10.1074/JBC.M113.517011

Wagner, S., Herrmannová, A., Hronová, V., Gunišová, S., Sen, N. D., Hannan, R. D., et al. (2020). Selective Translation Complex Profiling Reveals Staged Initiation and Co-translational Assembly of Initiation Factor Complexes. Mol. Cel. 79, 546-560. doi:10.1016/j.molcel.2020.06.004

Walker, S. E., Zhou, F., Mitchell, S. F., Larson, V. S., Valasek, L., Hinnebusch, A. G., et al. (2013). Yeast eIF4B Binds to the Head of the 40S Ribosomal Subunit and Promotes mRNA Recruitment through its N-Terminal and Internal Repeat Domains. RNA 19, 191-207. doi:10.1261/rna.035881.112

Yourik, P., Aitken, C. E., Zhou, F., Gupta, N., Hinnebusch, A. G., and Lorsch, J. R. (2017). Yeast eIF4A Enhances Recruitment of mRNAs Regardless of Their Structural Complexity. Elife 6, 1. doi:10.7554/eLife.31476

Zhou, F., Zhang, H., Kulkarni, S. D., Lorsch, J. R., and Hinnebusch, A. G. (2020). eIF1 Discriminates against Suboptimal Initiation Sites to Prevent Excessive uORF Translation Genome-wide. RNA 26, 419-438. doi:10.1261/ rna.073536.119

Zinshteyn, B., Rojas-Duran, M. F., and Gilbert, W. V. (2017). Translation Initiation Factor eIF4G1 Preferentially Binds Yeast Transcript Leaders Containing Conserved Oligo-Uridine Motifs. RNA 23, 1365-1375. doi:10.1261/ RNA.062059.117

Conflict of Interest: The authors declare that the research was conducted in the absence of any commercial or financial relationships that could be construed as a potential conflict of interest.

Publisher's Note: All claims expressed in this article are solely those of the authors and do not necessarily represent those of their affiliated organizations, or those of the publisher, the editors and the reviewers. Any product that may be evaluated in this article, or claim that may be made by its manufacturer, is not guaranteed or endorsed by the publisher.

Copyright (c) 2022 Stanciu, Luo, Funes, Galbokke Hewage, Kulkarni and Aitken. This is an open-access article distributed under the terms of the Creative Commons Attribution License (CC BY). The use, distribution or reproduction in other forums is permitted, provided the original author(s) and the copyright owner(s) are credited and that the original publication in this journal is cited, in accordance with accepted academic practice. No use, distribution or reproduction is permitted which does not comply with these terms. 